\title{
Dynamical Analysis of a Pest Management Model with Saturated Growth Rate and State Dependent Impulsive Effects
}

\author{
Wencai Zhao, ${ }^{1}$ Tongqian Zhang, ${ }^{1}$ Xinzhu Meng, ${ }^{2}$ and Yang Yang ${ }^{2}$ \\ ${ }^{1}$ College of Science, Shandong University of Science and Technology, Qingdao 266590, China \\ ${ }^{2}$ College of Information Science and Engineering, Shandong University of Science and Technology, Qingdao 266590, China \\ Correspondence should be addressed to Tongqian Zhang; zhangtongqian@sdust.edu.cn
}

Received 11 March 2013; Accepted 20 May 2013

Academic Editor: Tonghua Zhang

Copyright (c) 2013 Wencai Zhao et al. This is an open access article distributed under the Creative Commons Attribution License, which permits unrestricted use, distribution, and reproduction in any medium, provided the original work is properly cited.

\begin{abstract}
A new pest management mathematical model with saturated growth is proposed. The integrated pest management (IPM) strategy by introducing two state dependent pulses into the model is considered. Firstly, we analyze singular points of the model qualitatively and get the condition for focus point. Secondly, by using geometry theory of impulsive differential equation, the existence and stability of periodic solution of the system are discussed. Lastly, some examples and numerical simulations are given to illustrate our results.
\end{abstract}

\section{Introduction}

Forest diseases and insect pests are the most destructive natural disasters to forestry; they are always an important constraint to forestry production worldwide and cause major economic losses in forestry. For example, spruce budworm, mountain pine beetle, and gypsy moth have led to significant losses in Canadian forests [1]. Therefore, how to prevent the occurrence and spreading of forest diseases and insect pests and reduce the economic loss is always an important subject in theory and practice. Mathematical models have been established to study change rule of the number of forest pests; these models include ordinary differential equation (ODE) system, difference equation system, partial differential equation (PDE) system, and so forth. In order to control pests, integrated pest management (IPM) [2-4] including two measures will be taken: one is chemical control (spraying pesticide) and the other is biological control (releasing natural enemy). In the process of pest management, if the amount of pests is small, biological control will be implemented, and if the amount of pests is large, integrated pest control will be applied. Usually, system with sudden disturbance always leads to impulsive system. In the forest pest management system, human's interference can cause a sudden change of the number or density of pests. So impulsive differential equation (IDE) can be established to explain this phenomenon.
Many biological models based on time impulse [5-13] or one state impulse $[14,15]$ have been studied by many authors. The model with two state impulses is very rare. In $[16,17]$, two models with two state impulses are established to study the existence and the stability of periodic solution of the models, respectively, by Poincaré map and the Lambert $M$ function. In [18], a model with two state impulses is built up and the geometrical analysis of the model is made by Bendixson theorem of impulsive differential equations and the successor function. Motivated by $[15,17,18]$, we get the following reasonable model by introducing two state dependent impulses:

$$
\begin{aligned}
x^{\prime}(t) & =\frac{r x}{1+a x}-b x y, \quad x \neq h_{1}, h_{2}, \text { or } x=h_{1}, \quad y>y^{*}, \\
y^{\prime} & (t)=c x y-d y, \\
\Delta x & =0, \quad x=h_{1}, \quad y \leq y^{*}, \\
\Delta y & =\alpha, \quad \\
\Delta x & =-p x, \quad x=h_{2}, \\
\Delta y & =-q y+\tau, \quad
\end{aligned}
$$

where $\Delta x(t)=x\left(t^{+}\right)-x(t), \Delta y(t)=y\left(t^{+}\right)-y(t), x(t)$ is the density of pests at time $t$, and $y(t)$ is the density of natural enemies. $r x /(1+a x)$ is saturated growth rate, $b$ is 


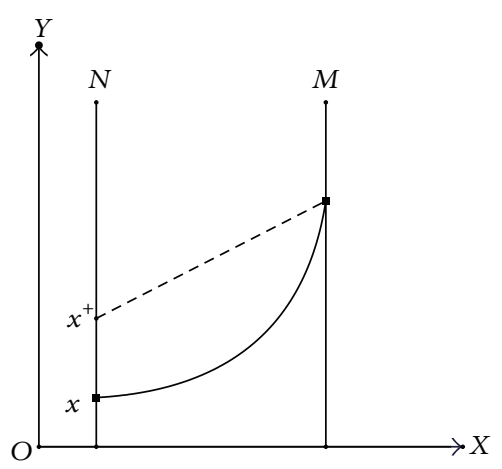

Figure 1: The successor function of system (2).

predation rate of natural enemies, and $c$ is the growth rate of natural enemies, after digestion and absorption. $d$ is the death rate of natural enemies. $h_{1}$ and $h_{2}$ are the threshold with slight damage and serious damage to the forest, respectively. $y^{*}$ is the ordinate of intersection of the line $x=h_{1}$ and $(r /(1+a x))-b y=0 . \alpha, \tau$ are the amount of natural enemies $y(t)$ released one time. $p$ and $q$ are death rates of pests and natural enemies due to pesticide, respectively. According to the biological significance, we only discuss $R_{+}^{2}=\{(x, y) \mid x \geq$ $0, y \geq 0\}$.

The paper is organized as follows. In Section 2, some definitions and theorems are given for the later use. In Section 3, the stability of system (1) without impulsive effect is investigated qualitatively. In Section 4, the existence and stability of periodic solution to system (1) are investigated using the successor function, Bendixson theorem, and stability theorem of periodic solution of impulsive differential equations. In Section 5, an example and some simulations are exerted to prove the results.

\section{Preliminaries}

We consider the state impulsive differential equation

$$
\begin{aligned}
& x^{\prime}(t)=P(x, y), \quad(x, y) \notin M\{x, y\}, \\
& y^{\prime}(t)=Q(x, y), \\
& \Delta x=\alpha(x, y), \quad(x, y) \in M\{x, y\}, \\
& \Delta y=\beta(x, y),
\end{aligned}
$$

where $M$ is one-dimensional line or curve in $R^{2} . M$ is called impulsive set, and the function $I$ is continuous mapping, $I(M)=N . I$ is called the impulse function, and $N$ is called the corresponding image set.

Definition 1. If there exists a point $P$ in the image set such that $f(P, T)=Q \in M\{x, y\}$, and the impulsive mapping $I(Q)=I(f(P, T))=P \in N$, then one calls $f(P, T)$ the periodic solution of system (2).

Definition 2. Function

$$
f(x)=f\left(x^{+}\right)-f(x)
$$

is called a successor function of the point $x$ (see Figure 1).
Theorem 3 (Bendixson theorem of impulsive differential equations). Assume $G$ is a Bendixson region of system (2); if $G$ does not contain any critical points of system (2), then system (2) has a closed orbit in $G$.

Theorem 4. Assume that, in continuous dynamic system (X, $\Pi)$, there exist two points $x_{1}, x_{2}$ in the pulse phase concentration such that the successor function $f\left(x_{1}\right)>0$ and $f\left(x_{2}\right)<0$; then there exists a point $P$ between the two points $x_{1}, x_{2}$ that makes $f(P)=0$; that is, the system has periodic solution.

Theorem 5 (see $[19,20])$. Consider the state impulsive differential equation

$$
\begin{aligned}
& x^{\prime}(t)=f(x, y), \quad \Phi(x, y) \neq 0, \\
& y^{\prime}(t)=g(x, y), \\
& \Delta x=\alpha(x, y), \quad \Phi(x, y)=0, \\
& \Delta y=\beta(x, y), \quad
\end{aligned}
$$

where the function $\Phi(x, y)=0$ is corresponding to the impulsive set $M$. The T-periodic solution $x=\phi(t), y=\varphi(t)$ of the model is orbitally asymptotically stable if the multiplier $\mu_{2}<1$ is calculated by the following formula:

$$
\begin{aligned}
\mu_{2}=\prod_{k=1}^{q} \Delta_{k} \exp \left[\int _ { 0 } ^ { T } \left(\frac{\partial f}{\partial x}(\phi(t), \varphi(t))\right.\right. \\
\left.\left.+\frac{\partial g}{\partial y}(\phi(t), \varphi(t))\right) d t\right],
\end{aligned}
$$

where

$$
\begin{aligned}
\Delta_{k}= & \left(f_{+}\left(\frac{\partial \beta}{\partial y} \cdot \frac{\partial \Phi}{\partial x}-\frac{\partial \beta}{\partial x} \cdot \frac{\partial \Phi}{\partial y}+\frac{\partial \Phi}{\partial x}\right)\right. \\
& \left.+g_{+}\left(\frac{\partial \alpha}{\partial x} \cdot \frac{\partial \Phi}{\partial y}-\frac{\partial \alpha}{\partial y} \cdot \frac{\partial \Phi}{\partial x}+\frac{\partial \Phi}{\partial y}\right)\right) \\
& \times\left(f \frac{\partial \Phi}{\partial x}+g \frac{\partial \Phi}{\partial y}\right)^{-1}
\end{aligned}
$$

and $f, g,(\partial \alpha / \partial x),(\partial \alpha / \partial y),(\partial \beta / \partial x),(\partial \beta / \partial y),(\partial \Phi / \partial x)$, $(\partial \Phi / \partial y)$ are calculated at the point $\left(\phi\left(t_{k}\right), \varphi\left(t_{k}\right)\right)$ and $f_{+}=$ $f\left(\phi\left(t_{k}^{+}\right), \varphi\left(t_{k}^{+}\right)\right), g_{+}=g\left(\phi\left(t_{k}^{+}\right), \varphi\left(t_{k}^{+}\right)\right)$.

\section{The Stability of System (1) without Impulsive Effect}

In this section, we consider system (1) without impulsive effect. Let $\alpha, \tau, p, q=0$; then we get the following system:

$$
\begin{gathered}
x^{\prime}(t)=\frac{r x}{1+a x}-b x y, \\
y^{\prime}(t)=y(-d+c x) .
\end{gathered}
$$


The system has two equilibrium points $E_{0}(0,0)$ and $E\left(x^{\mathrm{N}}\right.$, $\left.y^{\aleph}\right)$, where $x^{\aleph}=d / c$ and $y^{\aleph}=r c /(b(a d+c))$. Assume that the variational matrix of the system (7) is as follows:

$$
J=\left(\begin{array}{cc}
\frac{r}{(1+a x)^{2}}-b y & -b x \\
c y & c x-d
\end{array}\right) .
$$

At $E_{0}(0,0)$,

$$
J\left(E_{0}\right)=\left(\begin{array}{cc}
r & 0 \\
0 & -d
\end{array}\right) .
$$

Obviously, $E_{0}(0,0)$ is saddle point. At $E\left(x^{\aleph}, y^{\aleph}\right)$,

$$
J\left(E\left(x^{\aleph}, y^{N}\right)\right)=\left(\begin{array}{cc}
-\frac{r a c d}{(c+a d)^{2}} & -\frac{b d}{c} \\
\frac{r c^{2}}{b(c+a d)} & 0
\end{array}\right) .
$$

The characteristic equation of $J(E)$ satisfies $f(\lambda)=\lambda^{2}+p \lambda+$ $q=0$, where $p=\operatorname{racd} /(c+a d)^{2}, q=(r c d /(a d+c))>0$ and $\Delta=p^{2}-4 q=r c d\left[r a^{2} c d-4(a d+c)^{3}\right] /(a d+c)^{4}$. Analyse $\Delta ;$ we have the following conclusion:

(i) if $\Delta>0$, that is, $r a^{2} c d>4(a d+c)^{3}$, then $E\left(x^{\aleph}, y^{\aleph}\right)$ is stable node;

(ii) if $\Delta=0$, that is, $r a^{2} c d=4(a d+c)^{3}$, then $E\left(x^{\aleph}, y^{\aleph}\right)$ is stable critical node;

(iii) if $\Delta<0$, that is, $r a^{2} c d<4(a d+c)^{3}$, then $E\left(x^{\aleph}, y^{\aleph}\right)$ is stable focus.

Denote $\left(H_{1}\right): r a^{2} c d<4(a d+c)^{3}$; we have the following theorem.

Theorem 6. The positive equilibrium point of system (7) is stable focus provided $\left(H_{1}\right)$ is true.

Theorem 7. The solution of system (7) is bounded.

Proof. Let $(x(t), y(t))$ be solution of system (7) satisfied initial conditions $x\left(t_{0}\right)=x_{0}>0, y\left(t_{0}\right)=y_{0}>0$. From the second equation of system (7), it follows that $d y / d t>0$ for $x>d / c$. And there exists $T_{0}>t_{0}$, such that orbit $(x(t), y(t))$ intersects with isoclinic line $y=r /(b(1+a x))$ at point $\left(x\left(T_{0}\right), y\left(T_{0}\right)\right)$. Thus, we have $x(t)<x\left(T_{0}\right)$ for $t>T_{0}$. Let $K>x\left(T_{0}\right)$; we have $x(t)<K$ for $t>T_{0}$.

On the other hand, making three lines in the first quadrant, $\Gamma_{1}: x-K=0, \Gamma_{2}: y+(c / b) x-M=0$ and $\Gamma_{3}: y-M+(d / b)=$ 0 , we can acquire that

$$
\begin{aligned}
\frac{d \Gamma_{2}}{d t} & =\left[y(c x-d)+\frac{c}{b} x\left(\frac{r}{1+a x}-b y\right)\right]_{\Gamma_{2}} \\
& =\left[\frac{c r x}{b(1+a x)}+\frac{d c x}{b}\right]-M d .
\end{aligned}
$$

Then we have $d \Gamma_{2} / d t<0$ for $d / c<x<K$ and $M$ large enough. Similarly,

$$
\frac{d \Gamma_{3}}{d t}=y(c x-d)
$$

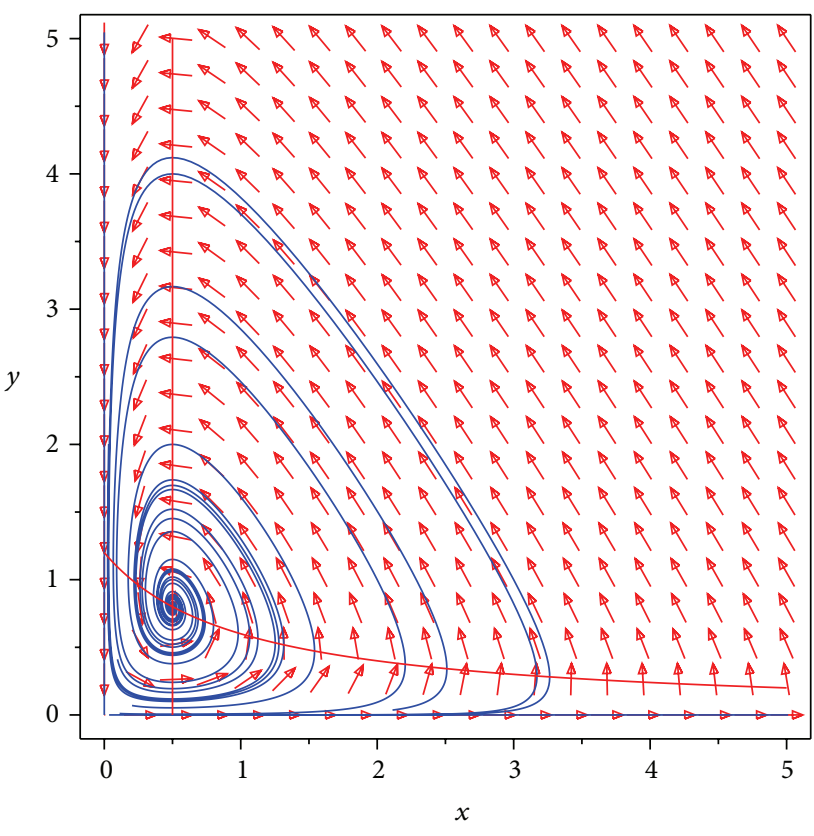

FigURE 2: Vector graph of system (7) with a stable focus, where $r=$ $0.6, a=1, b=0.5, c=0.8$, and $d=0.4$.

Then we have $d \Gamma_{3} / d t<0$ for $0<x<d / c$. According to the previous discussion, there exists an area $\Omega$ which is composed of $x=0, y=0, \Gamma_{1}, \Gamma_{2}, \Gamma_{3}$. For all initial points $\left(x\left(t_{0}\right), y\left(t_{0}\right)\right)$, $\exists T>0$, for $t>T$, we can acquire $(x(t), y(t)) \in \Omega$. Thus, the solution of system (7) satisfied initial conditions $\left(x\left(t_{0}\right), y\left(t_{0}\right)\right)$ is bounded.

Theorem 8. The positive equilibrium point of system (7) is globally asymptotically stable provided condition $\left(H_{1}\right)$ is true.

Proof. Firstly, we claim that the positive equilibrium point of system (7) is stable focus point and around it there does not exist closed rail line provided condition $\left(H_{1}\right)$ is true. In fact, let Dulac function be $B=1 / x y$; in regard to the system ( 7$)$, it follows that

$$
D=\frac{\partial(P B)}{\partial x}+\frac{(Q B)}{\partial y}=\frac{-r a}{y(1+a x)^{2}}<0 .
$$

Due to the Bendixson-Dulac theorem, around $E$, there does not exist closed rail line. Synthesize Theorems 6 and 7; therefore we have that the positive equilibrium point of system (7) is globally asymptotically stable provided condition $\left(H_{1}\right)$ is true.

Vector graph of system (7) with a stable focus can be seen in Figure 2.

\section{The Analysis of System (1) with State Dependent on Pulse}

4.1. The Existence of Periodic Solutions of the System. In this section, we will discuss the existence of periodic solution of system (1) by using the successor function defined in this 


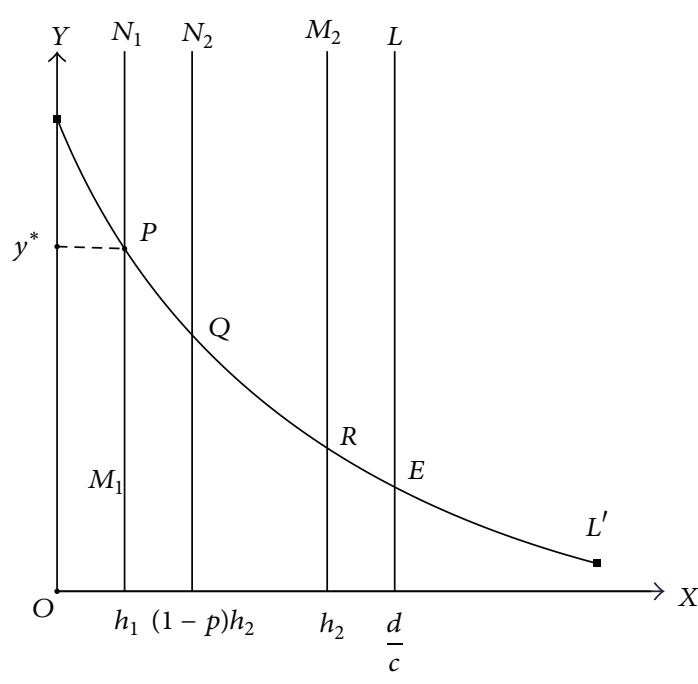

FIGURE 3: The structure graph of system (1).

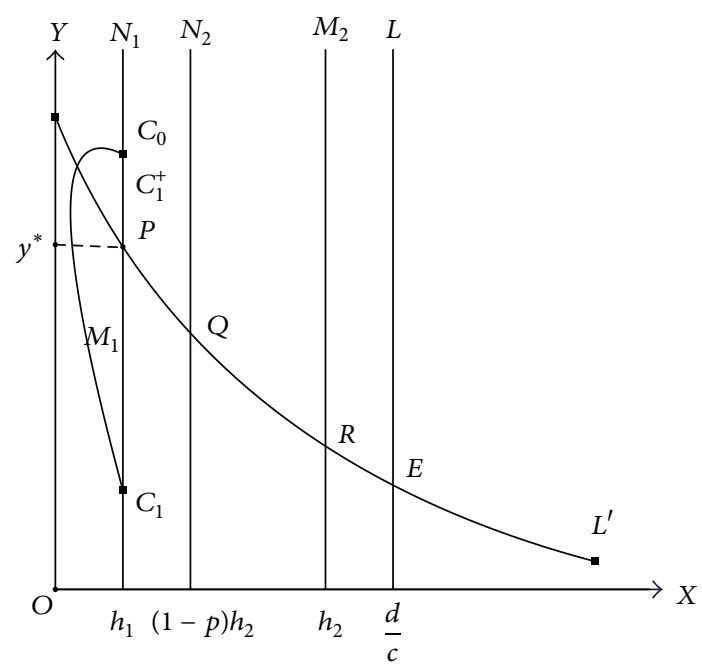

Figure 4: The orbit starting from the point $C_{0}$ above $P$ (Case 1 in Section 4.1.1).

paper and qualitative analysis. Let $\dot{x}=0, \dot{y}=0$; we know the curve $L^{\prime}: y=r /(b(1+a x))$ and $y$-axis are $X$-isoline and the line $L: x=d / c$ and $x$-axis are $Y$-isoline. From the previous discussion, we know $E\left(x^{\aleph}, y^{\aleph}\right)$ is a stable focus provided $\left(H_{1}\right)$ is true. Denote the first impulsive set as $M_{1}=\{(x, y) \mid x=$ $\left.h_{1}, 0 \leq y \leq y^{*}\right\}$, the second impulsive set as $M_{2}=\{(x, y) \mid$ $\left.x=h_{2}, y \geq 0\right\}$, the image set corresponding to set $M_{1}$ as $N_{1}=\left\{(x, y) \mid x=h_{1}, 0<y \leq y^{*}+\alpha\right\}$, and the image set corresponding to set $M_{2}$ as $N_{2}=\{(x, y) \mid x=(1-$ p) $h_{2}, y \geq \tau$. Let points $P, Q$, and $R$ be the intersection of line $L^{\prime}$ with lines $N_{1}, N_{2}$, and $M_{2}$, respectively. Due to the practical significance, in this paper we assume the set always lies in the left side of stable focus $E$; that is, $h_{1}<(1-p) h_{2}<h_{2}<d / c$. We will start our discussion about the existence of periodic

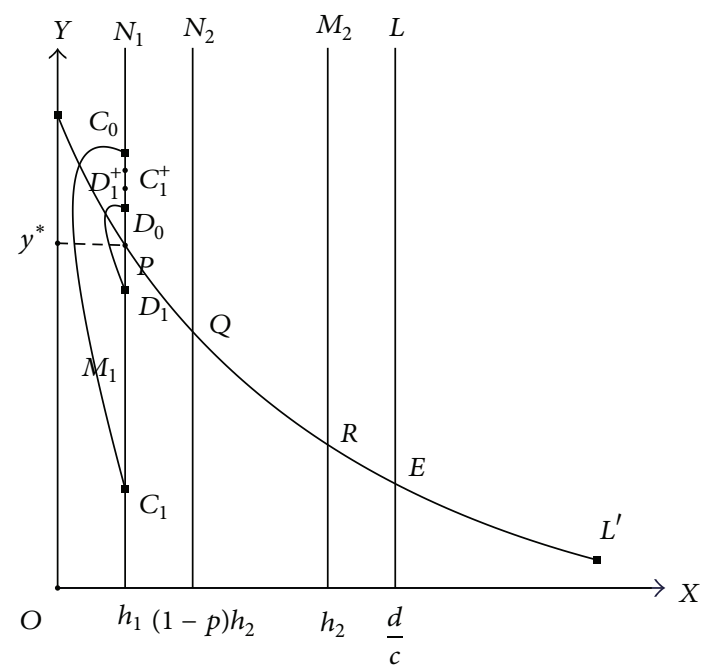

FIgURE 5: The orbit starting from the point $C_{0}$ (Case 2 in Section 4.1.1).

solution for (1) with the initial point $C_{0}\left(x_{c_{0}}, y_{c_{0}}\right)$. The structure graph of system (1) can be seen in Figure 3.

4.1.1. The Orbit Starting from the Point $C_{0}$ on $N_{1}$ above $P$. Starting from $C_{0}$, the orbit intersects with $M_{1}$ at point $C_{1}\left(x_{c_{1}}, y_{c_{1}}\right)$ and then produces pulse to the point $C_{1}^{\prime}\left(x_{c_{1}^{\prime}}, y_{c_{1}^{\prime}}\right)$ on $M_{1}$. According to (1), the following is got: $y_{C_{1}^{\prime}}=y_{C_{1}}+\alpha$. After $n$ time impulse, the point $C_{1}^{+}\left(x_{c_{1}^{+}}, y_{c_{1}^{+}}\right)$should satisfy $y^{*}<y_{C_{1}^{+}} \leq y^{*}+\alpha$. Thus, we only need to consider case $y^{*}<y_{C_{0}} \leq y^{*}+\alpha$, and three cases should be discussed.

Case 1. If the impulsive point $C_{1}^{+}$corresponding to $C_{1}$ is exactly $C_{0}$, thus, the curve $C_{0} C_{1} C_{1}^{+}$shall constitute a periodic orbit of (1) (see Figure 4).

Case 2. If the impulsive point $C_{1}^{+}$corresponding to $C_{1}$ is below $C_{0}$ on $N_{1}$, obviously, the successor function of $C_{0}$ satisfies $f\left(C_{0}\right)=y_{C_{1}^{+}}-y_{C_{0}}<0$. On the other hand, choose a point $D_{0}$ next to $P$ on $N_{1}$ (i.e., $\left|D_{0} P\right|<\varepsilon$ ). The orbit of (1) starting from point $D_{0}$ intersects with $M_{1}$ at point $D_{1}$ and then jumps to point $D_{1}^{+}$in the line $N_{1}$. Because $D_{0}$ is next to $P, D_{1}$ is next to $P$, and $y_{D_{1}^{+}}=y_{D_{1}}+\alpha$, thus, the successor function of $D_{0}$ satisfies $f\left(D_{0}\right)=y_{D_{1}^{+}}-y_{D_{0}}>0$. By Theorem 4 there exists a period solution (see Figure 5).

Case 3. If the impulsive point $C_{1}^{+}$corresponding to $C_{1}$ is above $C_{0}$ on $N_{1}$, obviously $f\left(C_{0}\right)>0$. On the other hand, the orbit starting from point $A\left(h_{1}, y^{*}+\alpha\right)$ intersects with $M_{1}$ at point $A_{1}$ and then jumps to point $A_{1}^{+}\left(h_{1}, y_{A_{1}^{+}}\right)$in the line $N_{1}$. According to system (1), the following is got: $y^{*}<y_{A_{1}^{+}}<$ $y^{*}+\alpha$; then the successor function of $A$ satisfies $f(A)<0$. Based on the previous discussion, according to Theorem 4 , there exists a periodic orbit of (1) (see Figure 6).

4.1.2. The Orbit Starting from the Point $Q$. Starting from $Q$, the orbit intersects with $M_{2}$ at point $C_{1}$ and then produces 


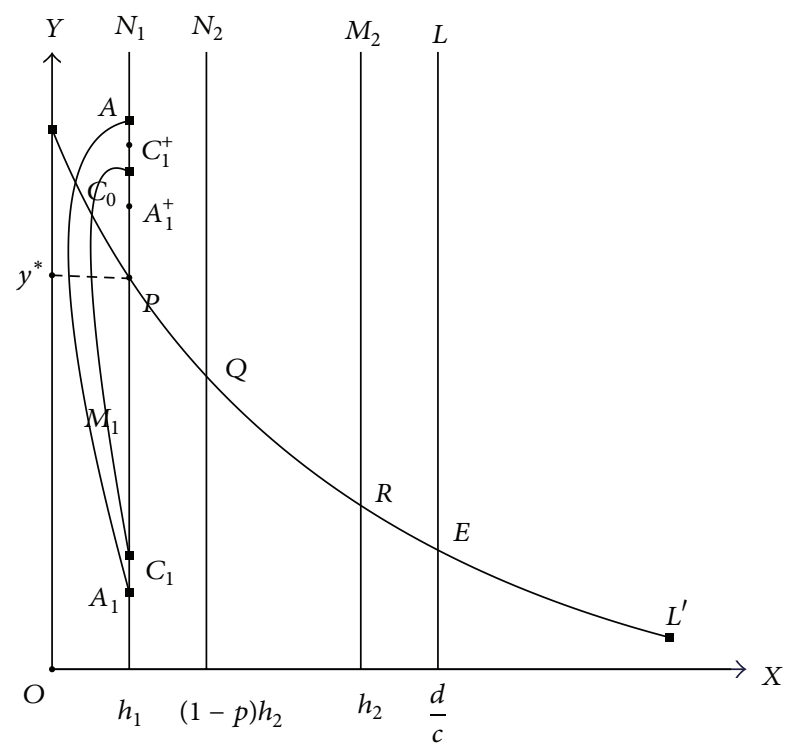

FIgure 6: The orbit starting from the point $C_{0}$ (Case 3 in Section 4.1.1).

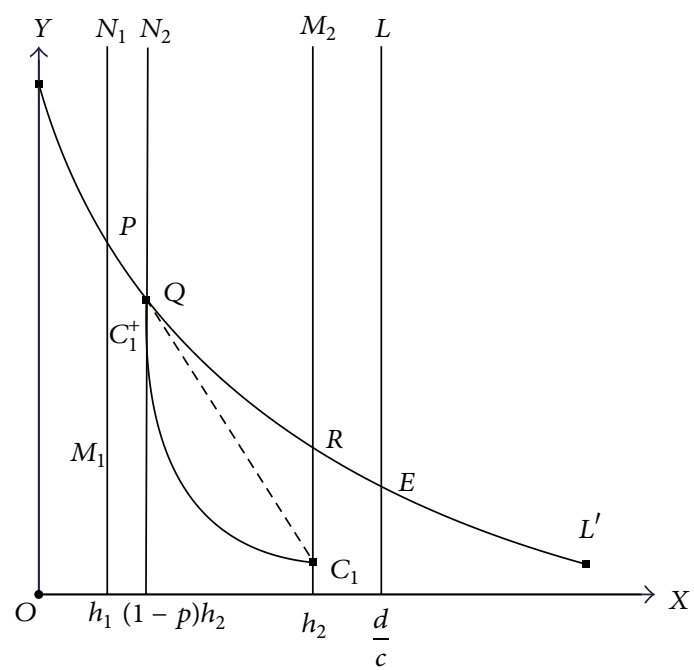

Figure 7: The orbit starting from the point $Q$ (Case 1 in Section 4.1.2).

pulse to the point $C_{1}^{+}$on $N_{2}$. According to (1), the following is got:

$$
\begin{gathered}
x_{C_{1}^{+}}=(1-p) h_{2}, \\
y_{C_{1}^{+}}=(1-q) y_{C_{1}}+\tau .
\end{gathered}
$$

By selecting $\tau$, three cases should be discussed.

Case 1. If the impulsive point $C_{1}^{+}$corresponding to $C_{1}$ is exactly $Q$, thus, the curve $Q C_{1} C_{1}^{+}$shall constitute a periodic orbit of (1) (see Figure 7).

Case 2. If the impulsive point $C_{1}^{+}$corresponding to $C_{1}$ is above $Q$ on $N_{2}$, thus, $f(Q)=y_{C_{1}^{+}}-y_{Q}>0$. Choose

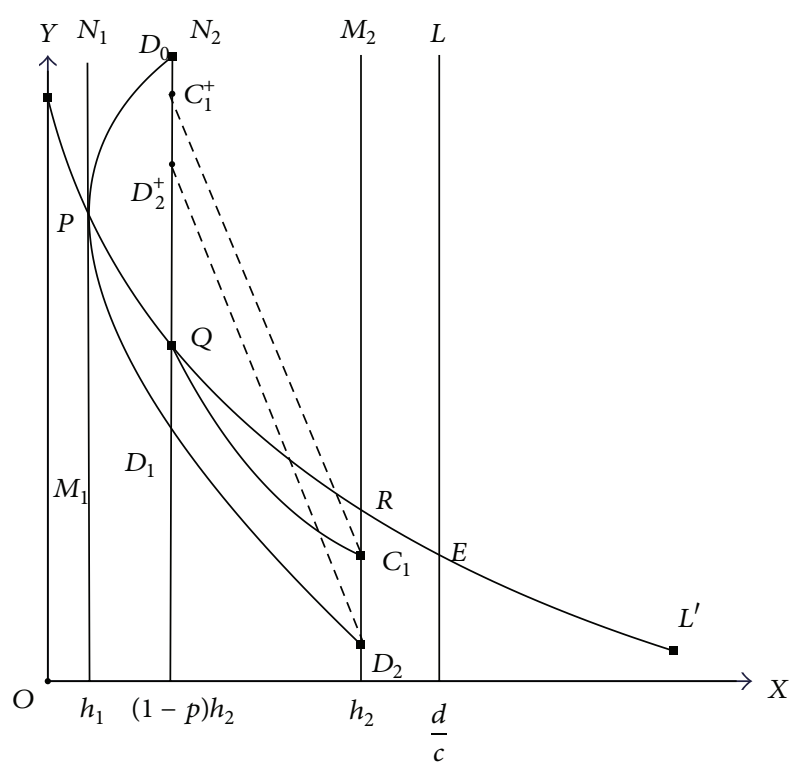

FIgURE 8: The orbit starting from the point $Q$ (Case 2 in Section 4.1.2).

a point $D_{0}$ above $C_{1}^{+}$in the straight line $N_{2}$. Only when it intersects with $N_{1}$ at $P$, can the orbit of (1) starting from point $D_{0}$ be vertical. After intersecting with $M_{2}$ at point $D_{2}$ through $N_{2}$ from left to right, it jumps to point $D_{2}^{+}$in the line $N_{2}$. According to the existence and uniqueness of impulsive differential equations, $D_{2}$ must be definitely below $C_{1}$ on $M_{2}$, and $D_{2}^{+}$must be below $C_{1}^{+}$on $N_{2}$; therefore the successor function of $D_{0}$ is $f\left(D_{0}\right)=y_{D_{2}^{+}}-y_{D_{0}}<0$. Based on the previous discussion, we can see that the region $\kappa$ surrounded by the closed curve $D_{0} P D_{1} D_{2} C_{1} C_{1}^{+} D_{0}$ is a positive invariant set of (1) and it contains no equilibrium point. According to Theorem 3 , there exists a periodic orbit of (1) in $\kappa$ (see Figure 8).

Case 3. If the impulsive point $C_{1}^{+}$corresponding to $C_{1}$ is below $Q$ on $N_{2}$, we shall have the successor function $f(Q)=$ $y_{C_{1}^{+}}-y_{Q}<0$.

In the meantime, take a point $D_{0}$ next to $B\left((1-p) h_{2}, 0\right)$ on $N_{2}\left(\left|D_{0} B\right| \ll \varepsilon\right.$.) Starting from $D_{0}$, the orbit intersects with $M_{2}$ at point $D_{1}$ and then produces pulse to the point $D_{1}^{+}$on $N_{2}$. According to (1), the following is got:

$$
\begin{gathered}
x_{D_{1}^{+}}=(1-p) h_{2}, \\
y_{D_{1}^{+}}=(1-q) y_{D_{1}}+\tau .
\end{gathered}
$$

Thus, $D_{1}^{+}$must be above $D_{0}$, and the successor function of $D_{0}$ is $f\left(D_{0}\right)=y_{D_{1}^{+}}-y_{D_{0}}>0$.

As a result, the region $\kappa$ surrounded by the closed curve $Q D_{0} D_{1} C_{1} Q$ involves a periodic solution of (1) (see Figure 9).

4.1.3. The Orbit Starting from the Point $C_{0}$ between the Second Impulsive Set $M_{2}$ and Its Image Set $N_{2}$. The orbit starting from point $C_{0}$, between line $N_{2}$ and line $M_{2}$, intersects with 


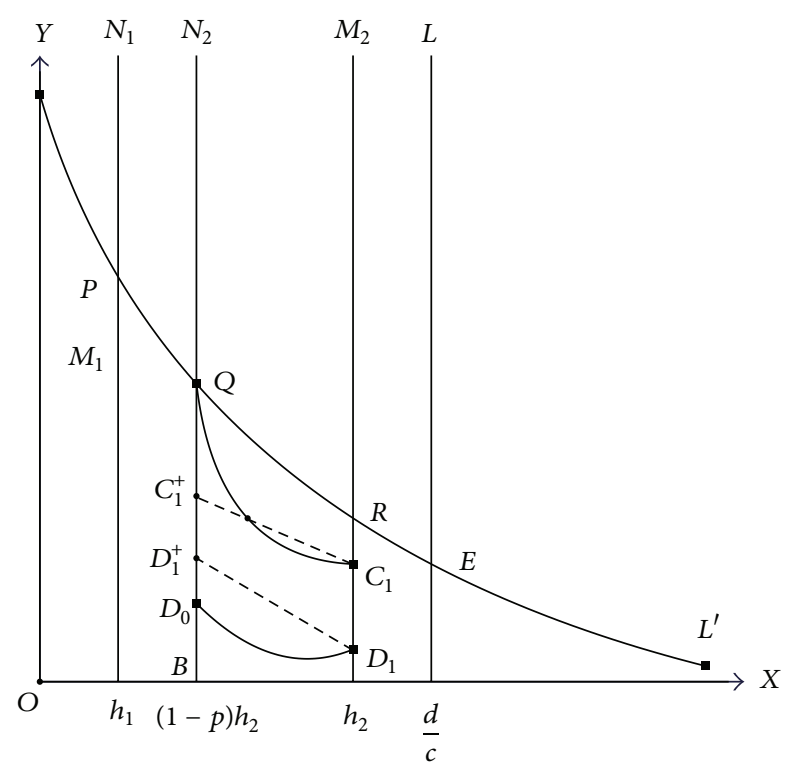

Figure 9: The orbit starting from the point $Q$ (Case 3 in Section 4.1.2).

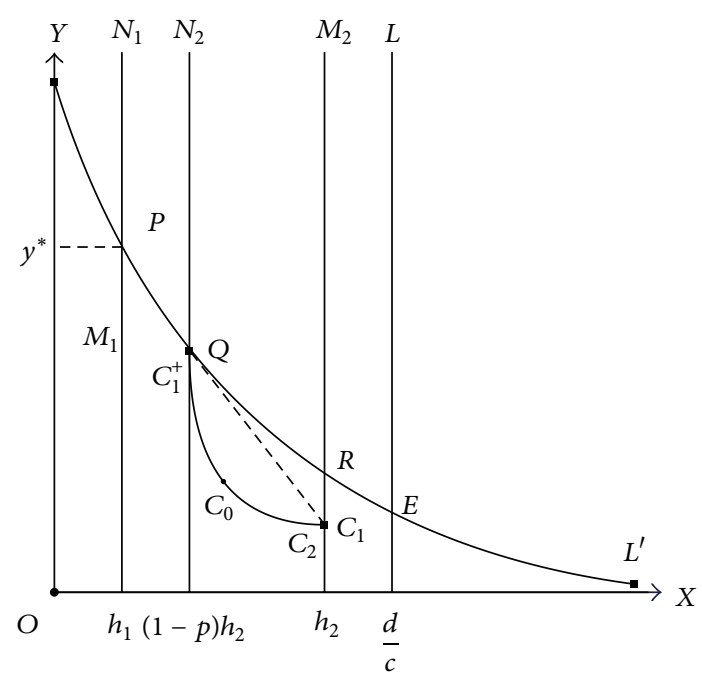

FIgURE 10: The orbit starting from the point $C_{0}$ (Case 1 (a) in Section 4.1.3).

line $M_{2}$ at $C_{1}$ and jumps onto point $C_{1}^{+}$on $N_{2}$. There are the following cases with changing $\tau$.

Case 1. If $C_{1}^{+}$on $N_{2}$ is exactly $Q$, the orbit from point $C_{1}^{+}$ moves to the point $C_{2}$ on $M_{2}$. For $C_{2}$, there are the following cases.

(a) If $C_{2}$ is exactly $C_{1}$, then the curve $C_{1} C_{1}^{+} C_{2}$ forms a periodic orbit of (1) (see Figure 10).

(b) If $C_{2}$ on $M_{2}$ is below $C_{1}$, the successor function of $C_{1}$ is $f\left(C_{1}\right)=y_{C_{2}}-y_{C_{1}}<0$.

In the meantime, take a point $D_{0}$ between $N_{2}$ and $M_{2}$ next to $x$-axis. After starting from $D_{0}$, the orbit hits

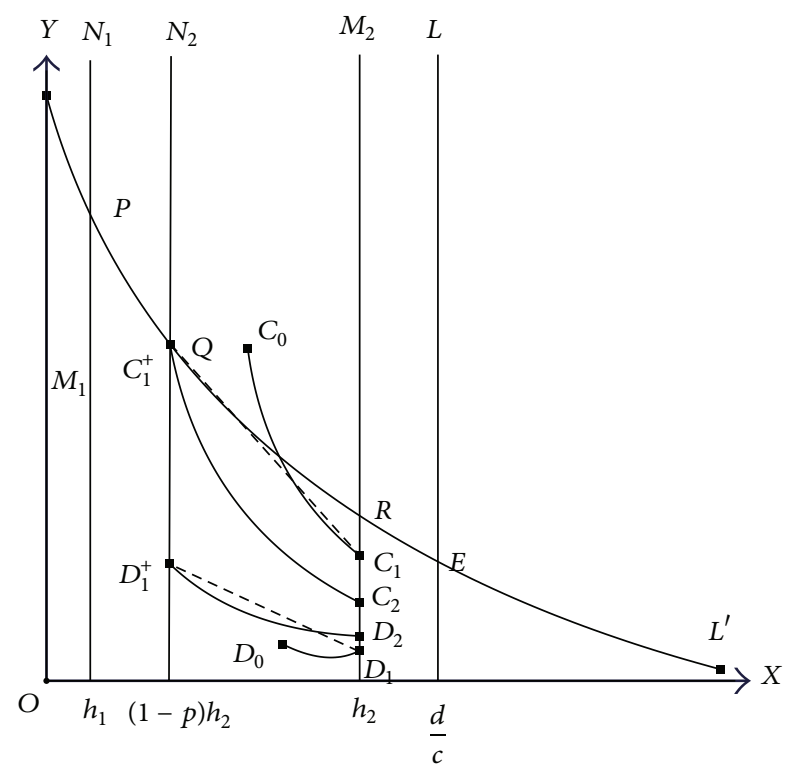

FIgURE 11: The orbit starting from the point $C_{0}$ (Case 1(b) in Section 4.1.3).

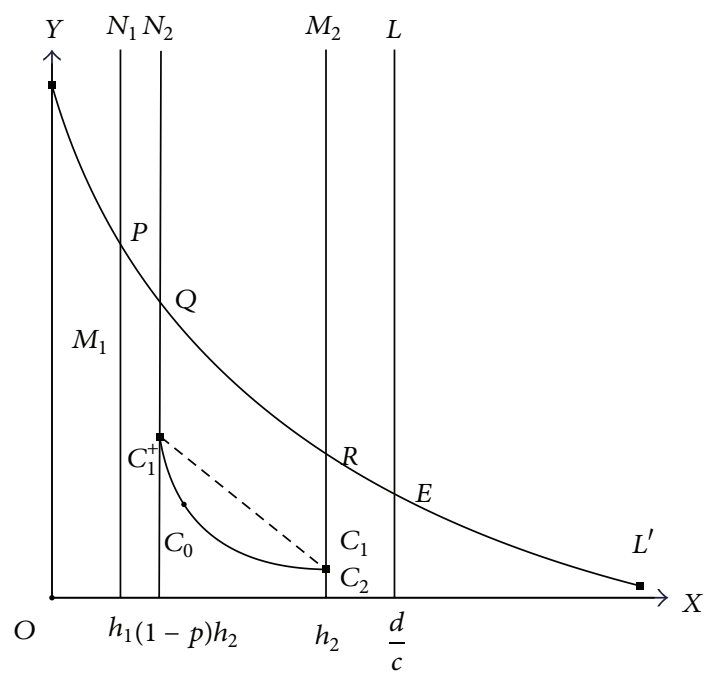

FIgure 12: The orbit starting from the point $C_{0}$ (Case 2 in Section 4.1.3).

the point $D_{1}$ on $M_{2}$, then jumps onto the point $D_{1}^{+}$on $N_{2}$, and then moves to the point $D_{2}$ on $M_{2}$. Obviously, $D_{2}$ is above $D_{1}$; thus, the successor function of $D_{1}$ is $f\left(D_{1}\right)=y_{D_{2}}-y_{D_{1}}>0$.

As a result, we get a periodic orbit in the region $\kappa$ encircled by the closed curve $C_{1} C_{1}^{+} D_{1}^{+} D_{1} C_{1}$ (see Figure 11).

(c) If $C_{2}$ on $M_{2}$ is above $C_{1}$, which does not exist periodic orbit, otherwise the curve $C_{1}^{+} C_{2}$ and $C_{0} C_{1}$ must be crossed, which leads to the conflict with the existence and uniqueness of solution to impulsive differential equations. 


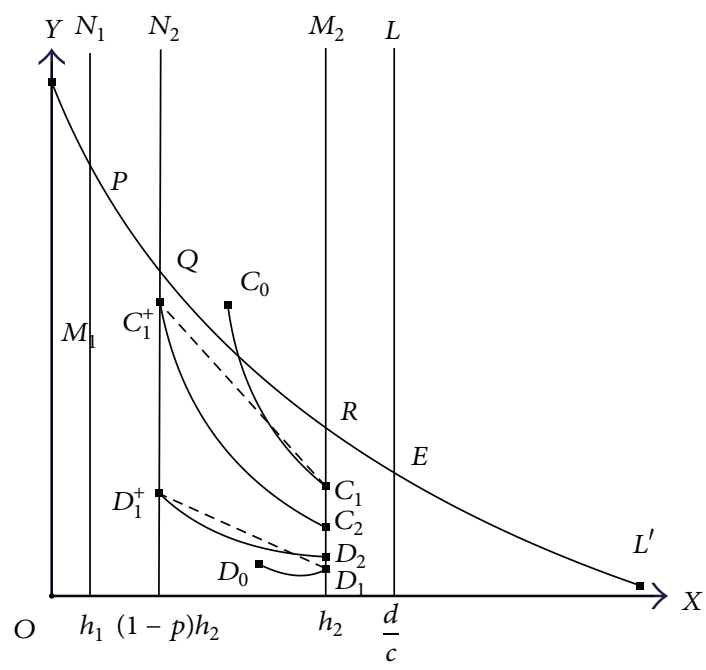

Figure 13: The orbit starting from the point $C_{0}$ (Case 2 in Section 4.1.3)

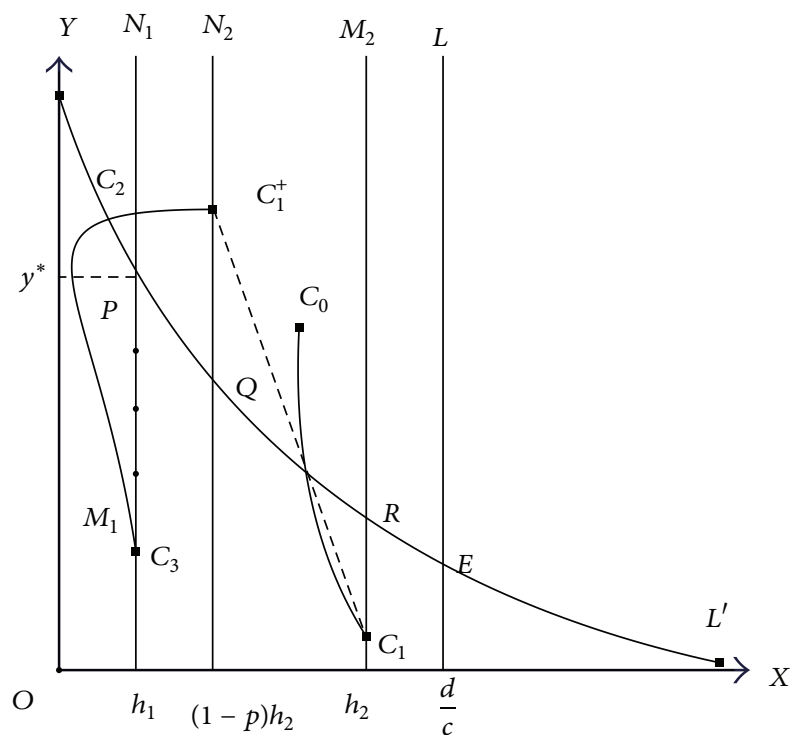

Figure 14: The orbit starting from the point $C_{0}$ (Case 3(a) in Section 4.1.3).

Case 2. If $C_{1}^{+}$on $N_{2}$ is below $Q$, the same conclusion can be got in the similar discussion previously mentioned (see Figures 12 and 13).

Case 3. If $C_{1}^{+}$on $N_{2}$ is above $Q$, for the orbit starting from $C_{1}^{+}$, there exist the following cases.

(a) If the orbit starting from $C_{1}^{+}$crosses $N_{1}$ from the right to the left and becomes vertical only when it goes through the line $L^{\prime}$, the point $C_{2}$ is the intersection of the orbit and $N_{1}$. The same conclusion can be got as the discussion in Section 4.1.1 (see Figure 14).

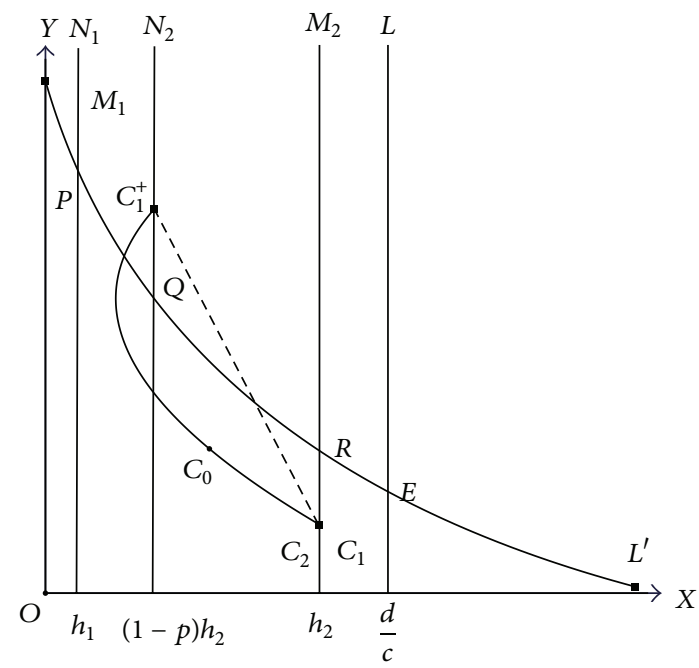

FIgURE 15: The orbit starting from the point $C_{0}$ (Case 3(b) in Section 4.1.3)

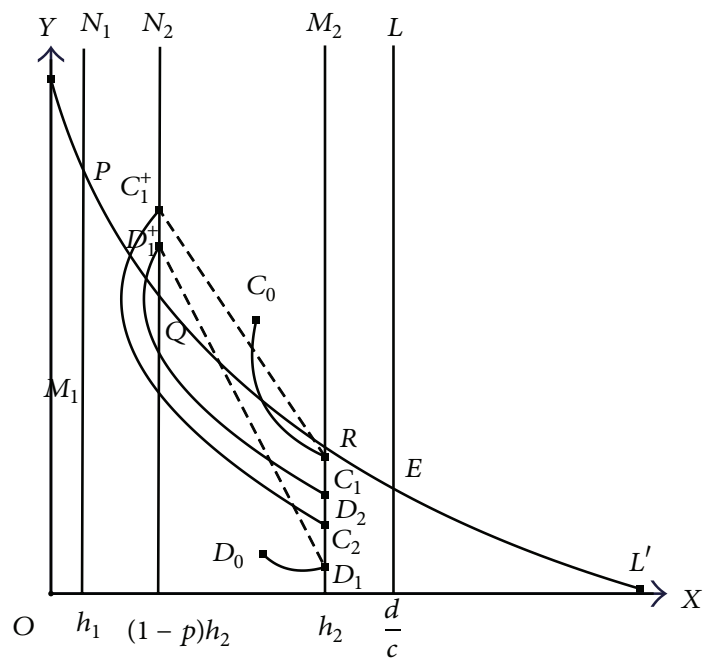

FIgure 16: The orbit starting from the point $C_{0}$ (Case 3(b) in Section 4.1.3).

(b) The orbit starting from $C_{1}^{+}$becomes vertical only when it crosses $P Q$, and then it moves on automatically to $C_{2}$ on $M_{2}$, which is exactly $C_{1}$, or below $C_{1}$, or above $C_{1}$.

If $C_{2}$ on $M_{2}$ is exactly $C_{1}$, then the curve $C_{1} C_{1}^{+} C_{2}$ forms a periodic orbit (see Figure 15).

If $C_{2}$ on $M_{2}$ is below $C_{1}$, then the successor function of $C_{1}$ is $f\left(C_{1}\right)=y_{C_{2}}-y_{C_{1}}<0$.

On the other hand, take a point $D_{0}$ between $N_{2}$ and $M_{2}$ near to $x$-axis, and the orbit starting from $D_{0}$ hits the point $D_{1}$ on $M_{2}$, then jumps onto the point $D_{1}^{+}$on $N_{2}$, and then returns to the point $D_{2}$ on $M_{2}$. 




(a) Phase diagram of system (24)

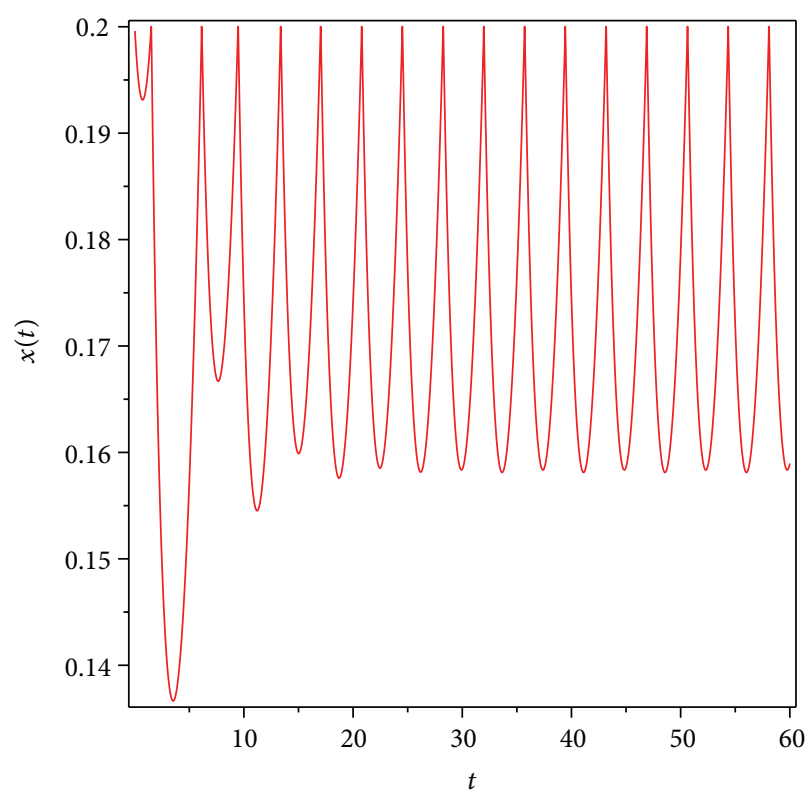

(b) Time series of system (24)

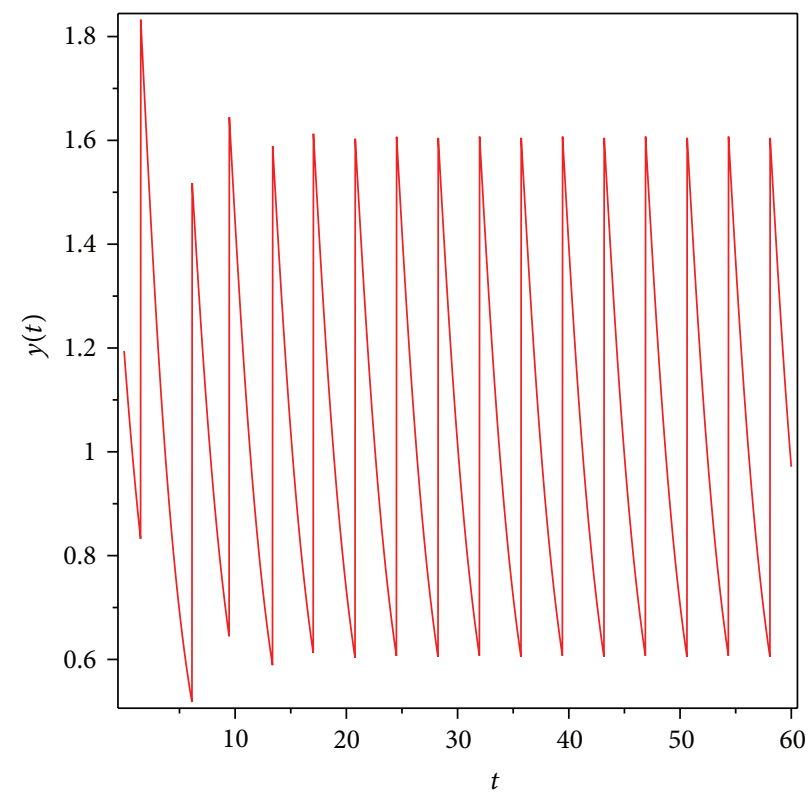

(c) Time series of system (24)

FIGURE 17: Illustration of basic behavior of system (24).

Obviously, $D_{2}$ on $M_{2}$ is above $D_{1}$. Thus, the successor function of $D_{1}$ is $f\left(D_{1}\right)=y_{D_{2}}-y_{D_{1}}>0$.

Based on the previous discussion above, there exists a periodic orbit in the region $\kappa$ encircled $D_{1} C_{1} C_{1}^{+} C_{2}$ and $C_{1}^{+} D_{1}^{+} D_{2} C_{2}$ (see Figure 16).

(c) If $C_{2}$ on $M_{2}$ is above $C_{1}$, which does not exist periodic orbit, the reason is the same as in Case $1(\mathrm{c})$ in Section 4.1.3.

\subsection{The Stability Analysis of Periodic Solutions of the System (1)}

4.2.1. The Stability of Periodic Solution about Impulsive Set $M_{1}$

Theorem 9. Let $x=\phi(t), y=\varphi(t)$ be the periodic solution of system $(1)$, and $\phi(0)=h_{1}, \varphi(0)=\varphi_{0}$; if $\varphi_{0}<\left(r / b\left(1+a h_{1}\right)\right)+\alpha$, the periodic solution is stable. 


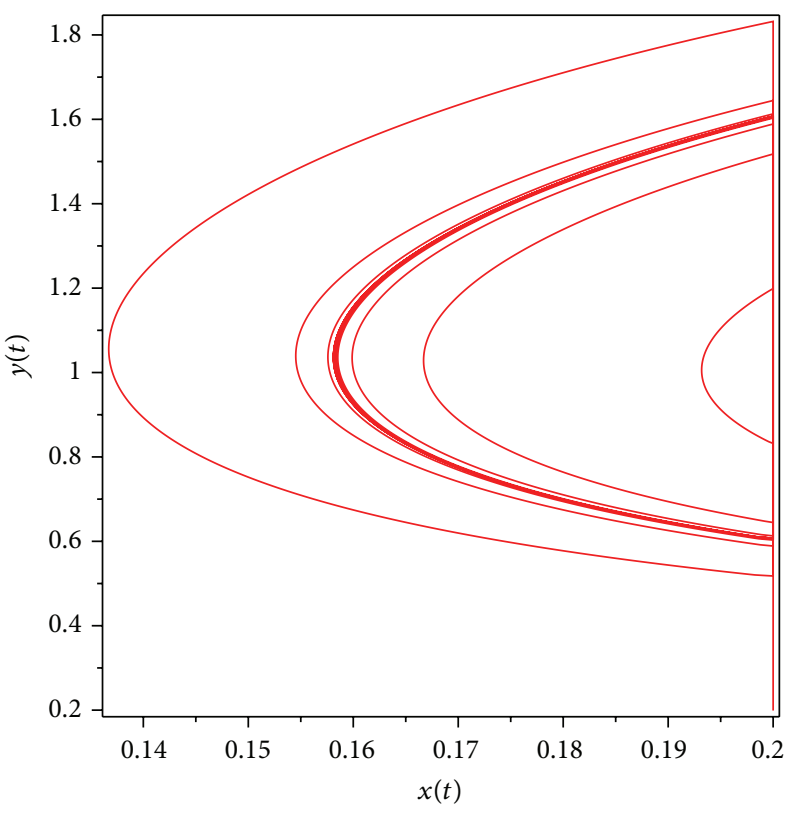

(a) Phase diagram of system (24)

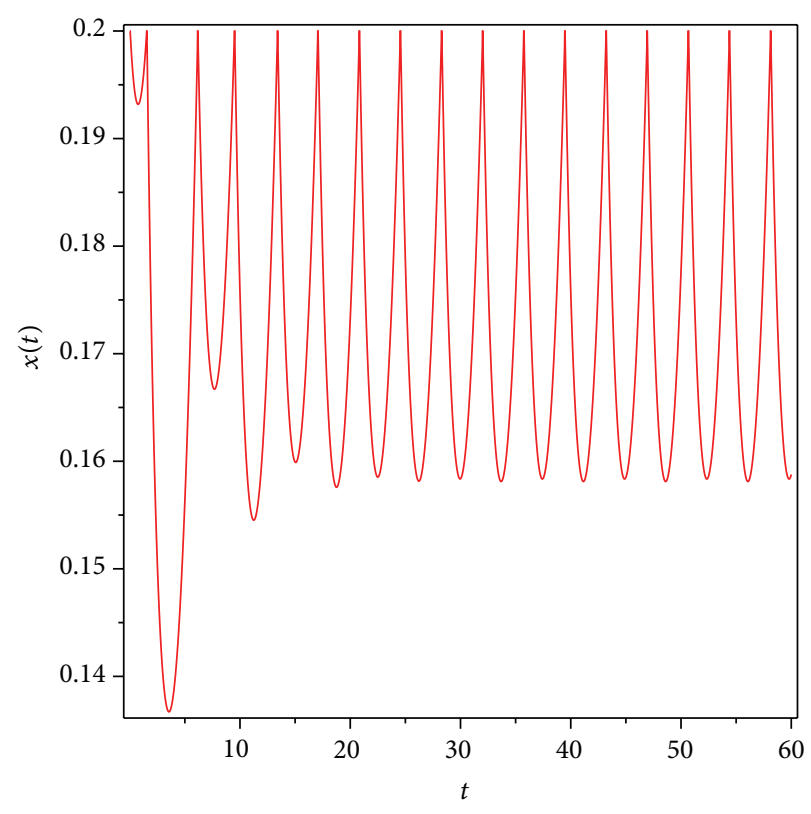

(b) Time series of system (24)

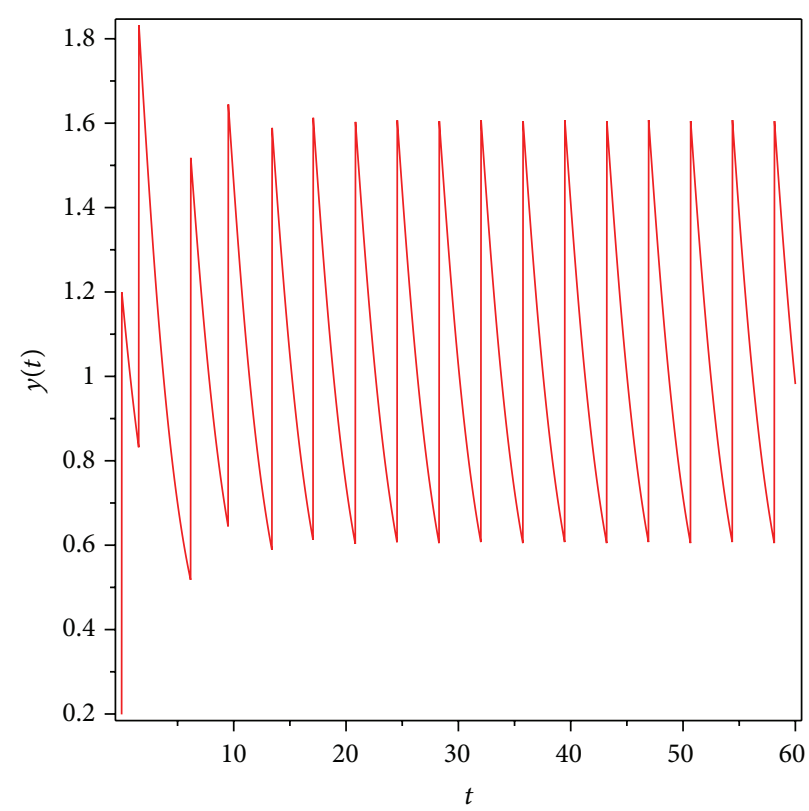

(c) Time series of system (24)

FIGURE 18: Illustration of basic behavior of system (24).

Proof. Let

$$
\begin{gathered}
f(x, y)=\frac{r x}{1+a x}-b x y, \\
g(x, y)=y(-d+c x), \\
\alpha(x, y)=0, \quad \beta(x, y)=\alpha, \\
\Phi(x, y)=x-h_{1},
\end{gathered}
$$

and $(\phi(0), \varphi(0))=\left(h_{1}, \varphi_{0}\right),(\phi(T), \varphi(T))=\left(x_{1}, y_{1}\right),\left(\phi\left(T^{+}\right)\right.$, $\left.\varphi\left(T^{+}\right)\right)=\left(x_{1}, y_{1}+\alpha\right)=\left(h_{1}, \varphi_{0}\right)$; one gets

$$
\begin{array}{rlrl}
\frac{\partial f}{\partial x}=\frac{r}{(1+a x)^{2}}-b y, & \frac{\partial g}{\partial y}=c x-d, \\
\frac{\partial \alpha}{\partial x}=0, & \frac{\partial \alpha}{\partial y}=0, & \frac{\partial \beta}{\partial x}=0, \\
\frac{\partial \beta}{\partial y}=0, & \frac{\partial \Phi}{\partial x}=1, & \frac{\partial \Phi}{\partial y}=0 .
\end{array}
$$




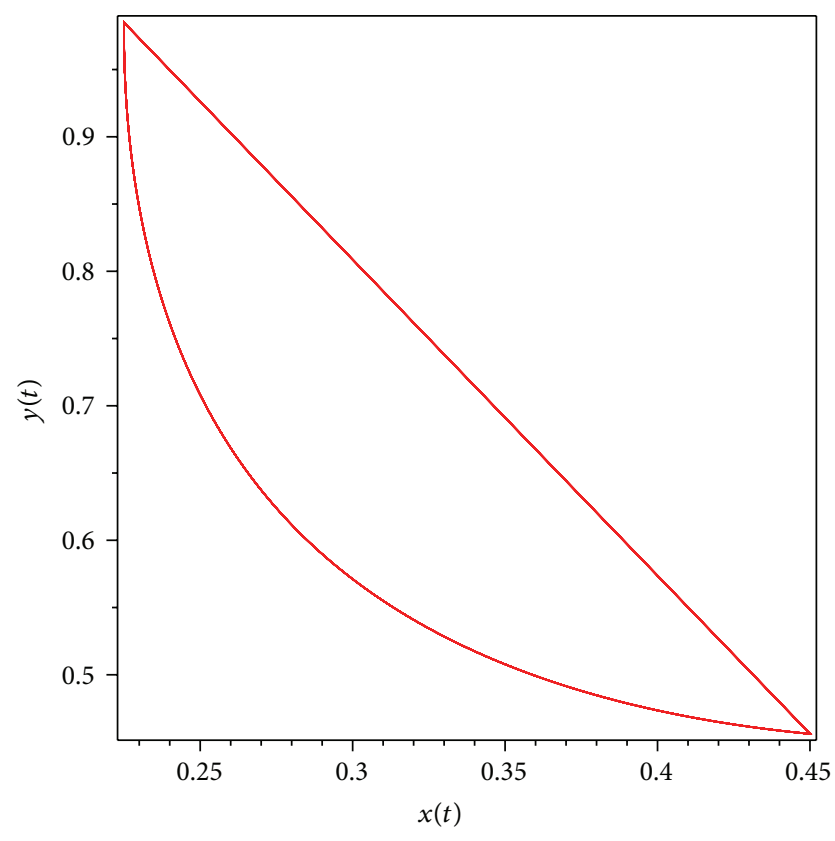

(a) Phase diagram of system (24)

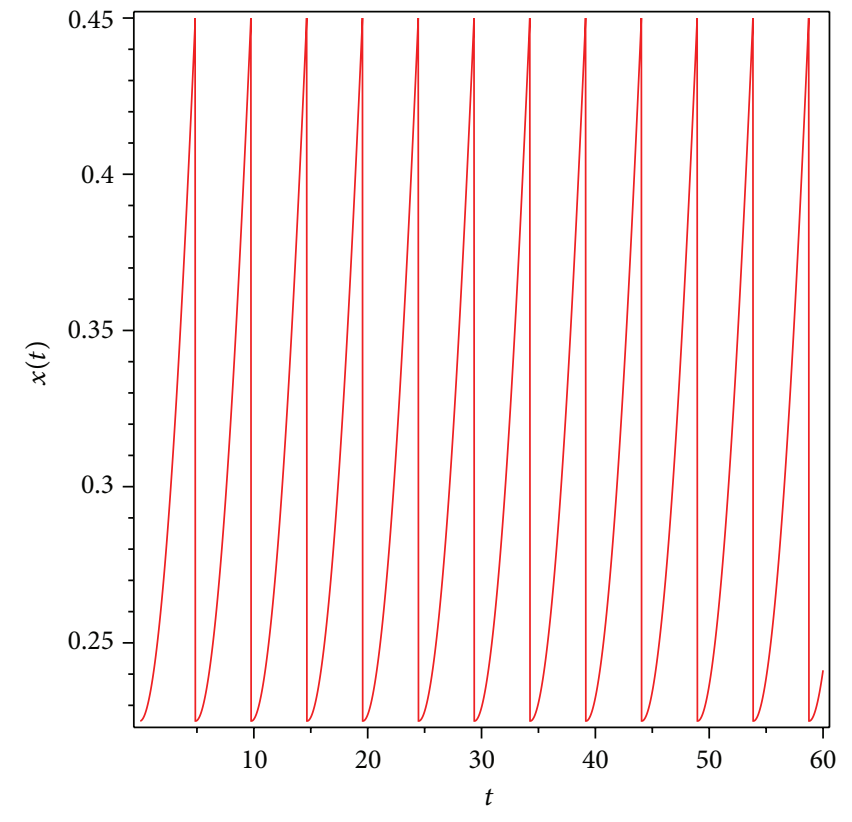

(b) Time series of system (24)

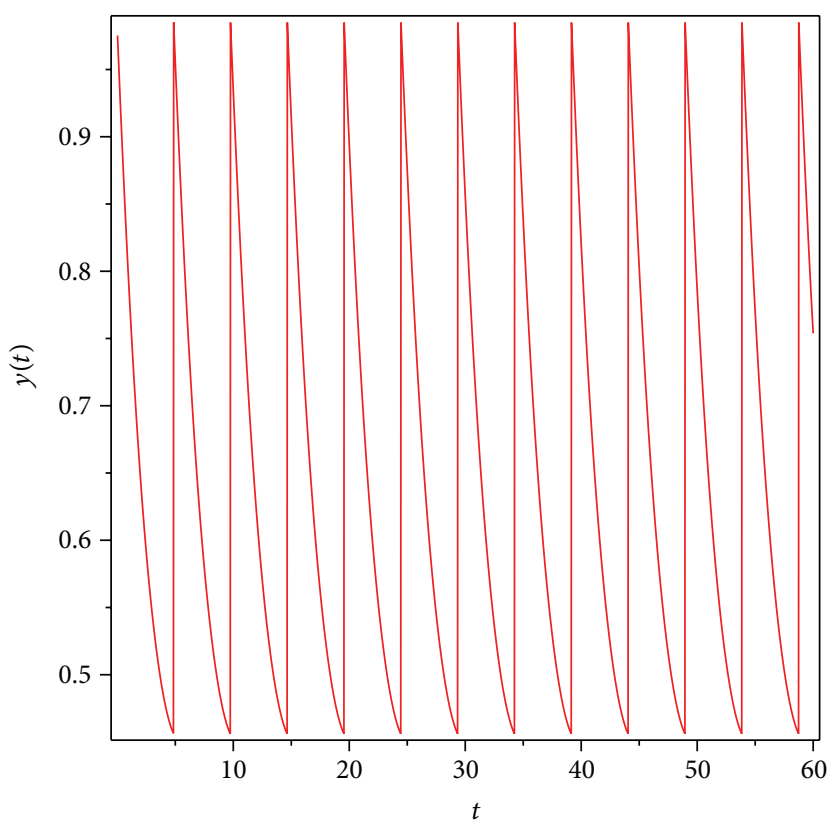

(c) Time series of system (24)

FIGURE 19: Illustration of basic behavior of system (24).

According to Theorem 5, we have

$$
\begin{aligned}
\Delta_{1} & =\frac{f\left(\phi\left(T^{+}\right), \varphi\left(T^{+}\right)\right)}{f(\phi(T), \varphi(T))}=\frac{f\left(h_{1}, \varphi_{0}\right)}{f\left(h_{1}, \varphi_{0}-\alpha\right)} \\
& =\frac{\left(r h_{1} /\left(1+a h_{1}\right)\right)-b h_{1} \varphi_{0}+b h_{1} \alpha-b h_{1} \alpha}{\left(r h_{1} /\left(1+a h_{1}\right)\right)-b h_{1}\left(\varphi_{0}-\alpha\right)} \\
& =1-\frac{b \alpha}{\left(r /\left(1+a h_{1}\right)\right)-b\left(\varphi_{0}-\alpha\right)}
\end{aligned}
$$

$$
\begin{aligned}
\mu_{2} & =\Delta_{1} \exp \int_{0}^{T}\left[\frac{\partial f}{\partial x}(\phi(t), \varphi(t))+\frac{\partial g}{\partial y}(\phi(t), \varphi(t))\right] d t \\
& =\Delta_{1} \exp \int_{0}^{T}\left[\frac{r}{(1+a \phi)^{2}}-b \varphi+c \phi-d\right] d t \\
& <\Delta_{1} \exp \int_{0}^{T}\left[\frac{r}{1+a \phi}-b \varphi+c \phi-d\right] d t
\end{aligned}
$$




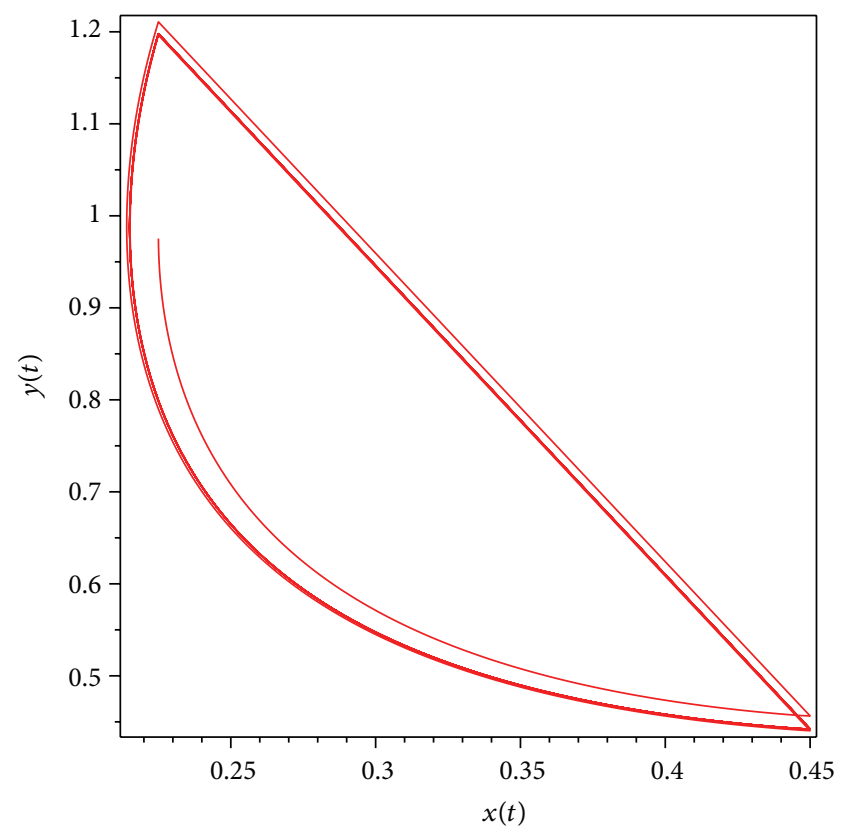

(a) Phase diagram of system (24)

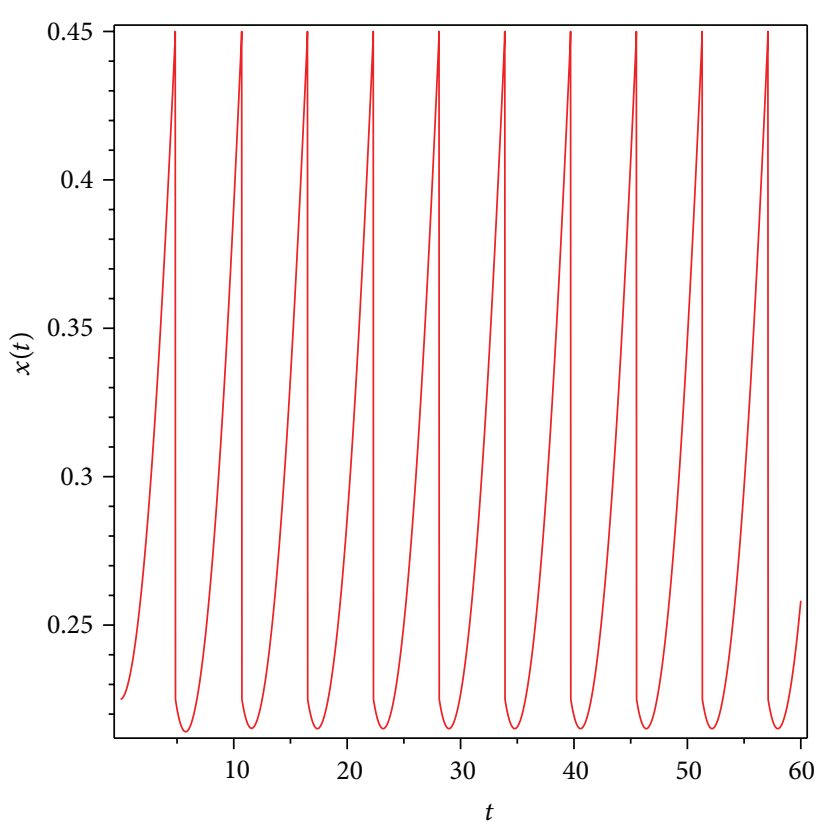

(b) Time series of system (24)

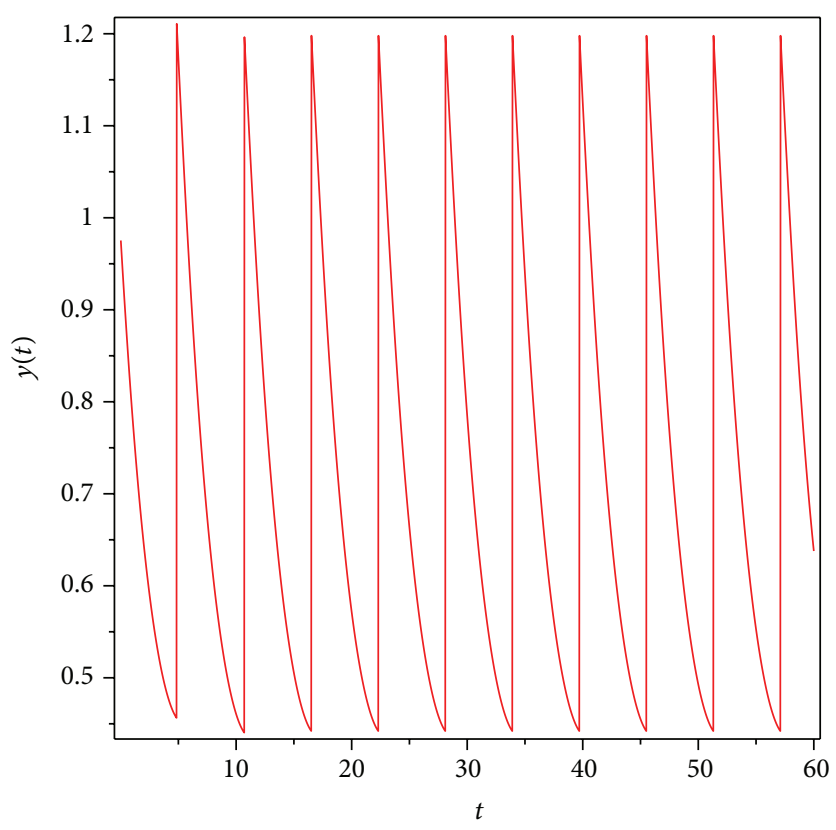

(c) Time series of system (24)

FIGURE 20: Illustration of basic behavior of system (24).

$$
\begin{aligned}
& =\Delta_{1} \exp \left[\int_{0}^{T} \frac{1}{\phi(t)} d \phi(t)+\int_{0}^{T} \frac{1}{\varphi(t)} d \varphi(t)\right] \\
& =\Delta_{1} \frac{\varphi_{0}-\alpha}{\varphi_{0}} \\
& =\left(1-\frac{b \alpha}{\left(r /\left(1+a h_{1}\right)\right)-b\left(\varphi_{0}-\alpha\right)}\right) \frac{\varphi_{0}-\alpha}{\varphi_{0}} .
\end{aligned}
$$

Obviously, if $\left(r /\left(1+a h_{1}\right)\right)-b\left(\varphi_{0}-\alpha\right)>0$, that is, $\varphi_{0}<$ $\left(r / b\left(1+a h_{1}\right)\right)+\alpha$, we have $\mu_{2}<1$. Thus the periodic solution to the system (1) is stable. This completes the proof.

\subsubsection{The Stability of Periodic Solution about Impulsive Set $M_{2}$}

Theorem 10. Let $x=\phi(t), y=\varphi(t)$ be the periodic solution of system $(1)$, and $(\phi(0), \varphi(0))=\left((1-p) h_{2}, \varphi_{0}\right)$; if $\varphi_{0}<$ $\min \left\{\left(r(1-q) / b\left(1+a h_{2}\right)\right)+\tau,(1 / b q)\left(b \tau-\left(a p(1-q) r h_{2} /(1+\right.\right.\right.$ 


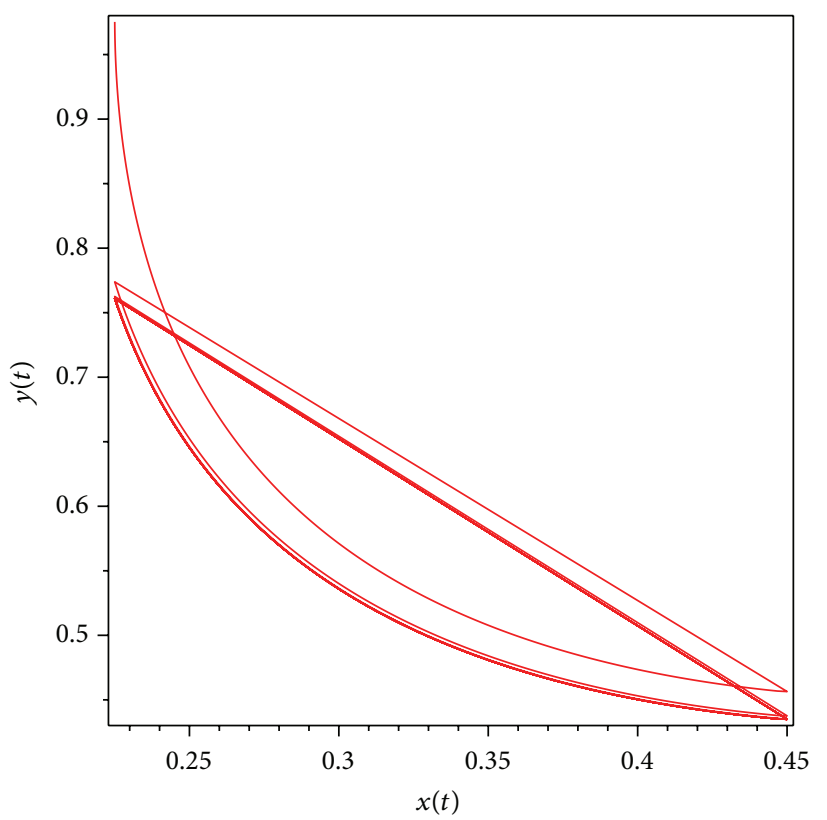

(a) Phase diagram of system (24)

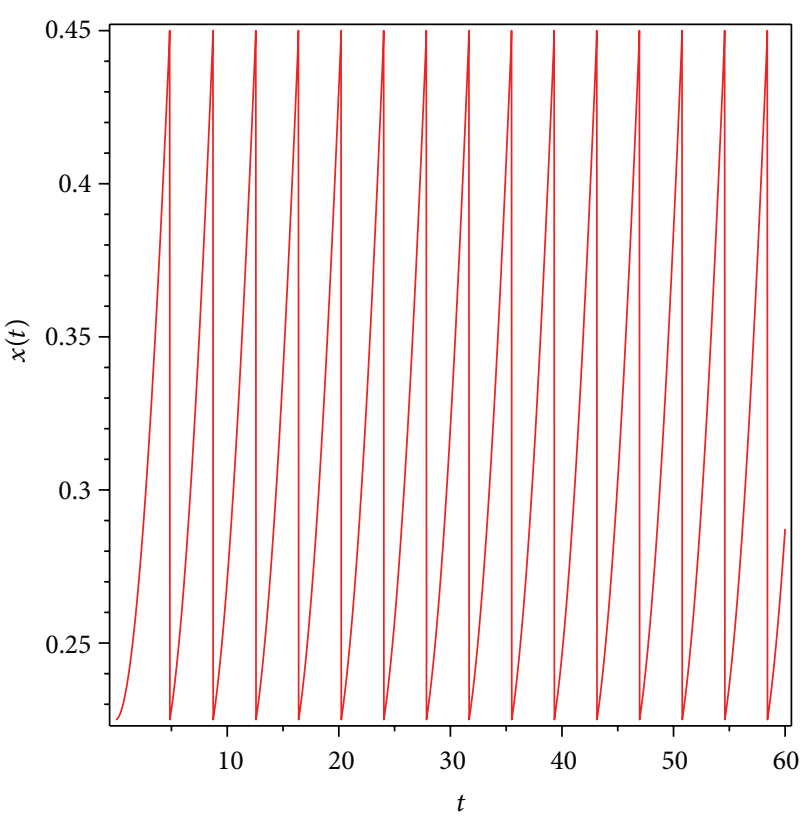

(b) Time series of system (24)

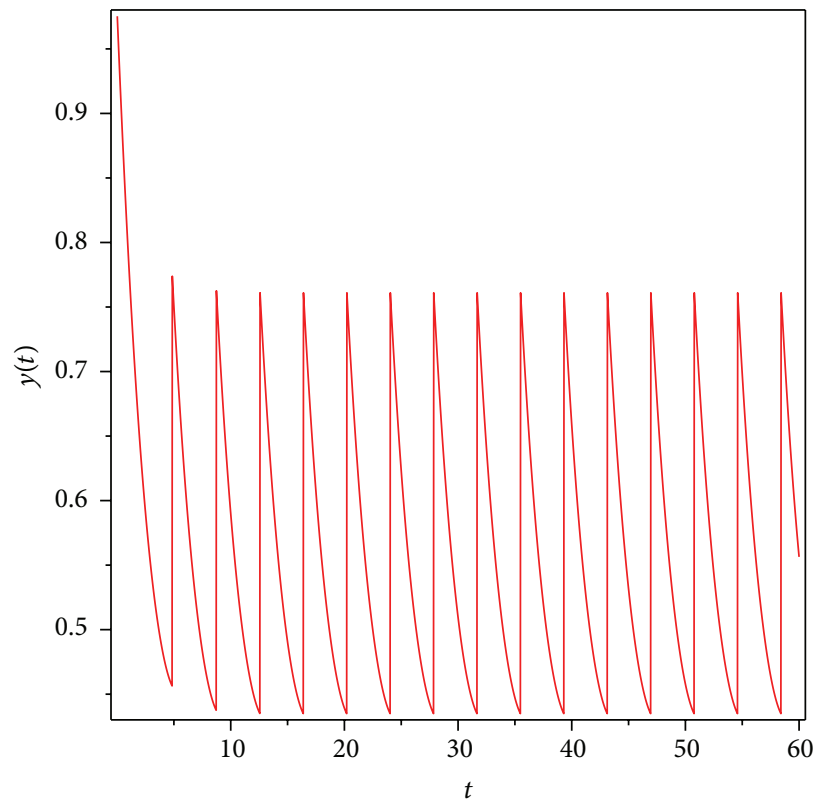

(c) Time series of system (24)

FIGURE 21: Illustration of basic behavior of system (24).

$\left.\left.\left.\left.a h_{2}\right)\left(1+a(1-p) h_{2}\right)\right)\right)\right\}$ or $\varphi_{0}>\max \left\{\left(r(1-q) / b\left(1+a h_{2}\right)\right)+\right.$ $\left.\tau,(1 / b q)\left(b \tau-\left(a p(1-q) r h_{2} /\left(1+a h_{2}\right)\left(1+a(1-p) h_{2}\right)\right)\right)\right\}$, the periodic solution to the system (1) is stable.

Proof. Let $x=\phi(t), y=\varphi(t)$ be the $T$ periodic solution of system (1), and

$$
\begin{aligned}
& f(x, y)=\frac{r x}{1+a x}-b x y, \\
& g(x, y)=y(-d+c x),
\end{aligned}
$$

$$
\begin{gathered}
\alpha(x, y)=-p x, \quad \beta(x, y)=-q y+\tau, \\
\Phi(x, y)=x-h_{2},
\end{gathered}
$$

$\left(\phi\left(T^{+}\right), \varphi\left(T^{+}\right)\right)=((1-p) \phi(T),(1-q) \varphi(T)+\tau),(\phi(T), \varphi(T))=$ $\left(h_{2},\left(\varphi_{0}-\tau\right) /(1-q)\right)$; one gets

$$
\frac{\partial f}{\partial x}=\frac{r}{(1+a x)^{2}}-b y, \quad \frac{\partial g}{\partial y}=c x-d,
$$




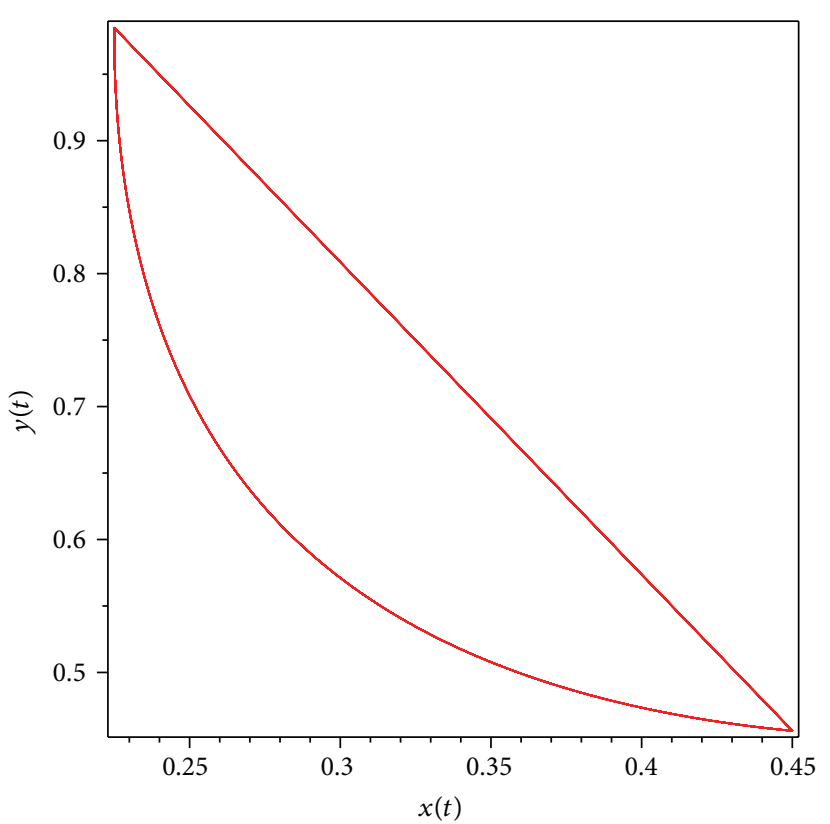

(a) Phase diagram of system (24)

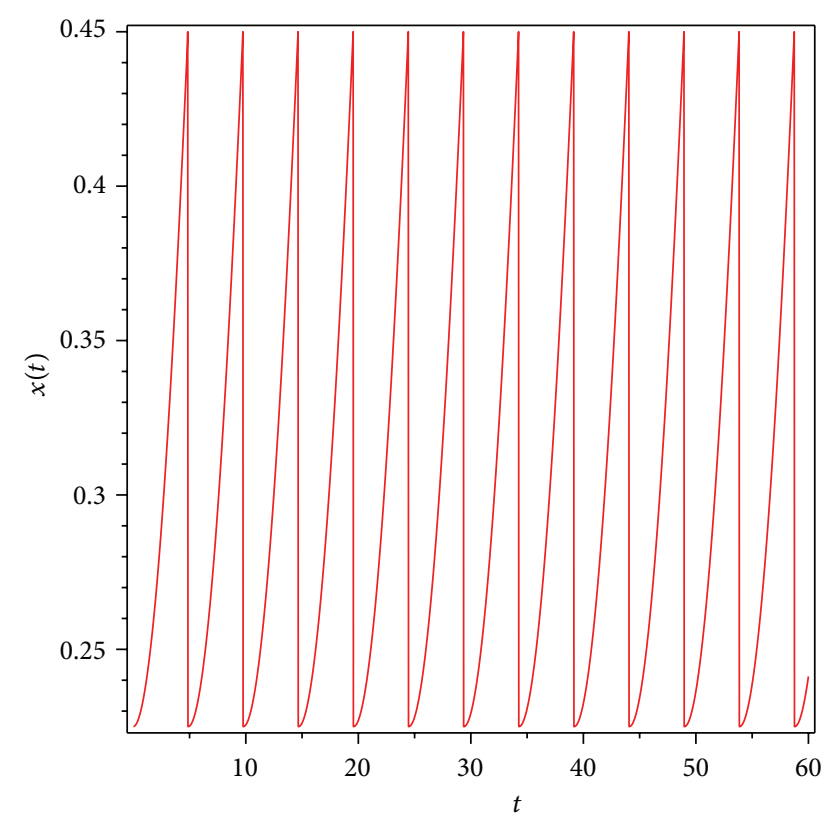

(b) Time series of system (24)

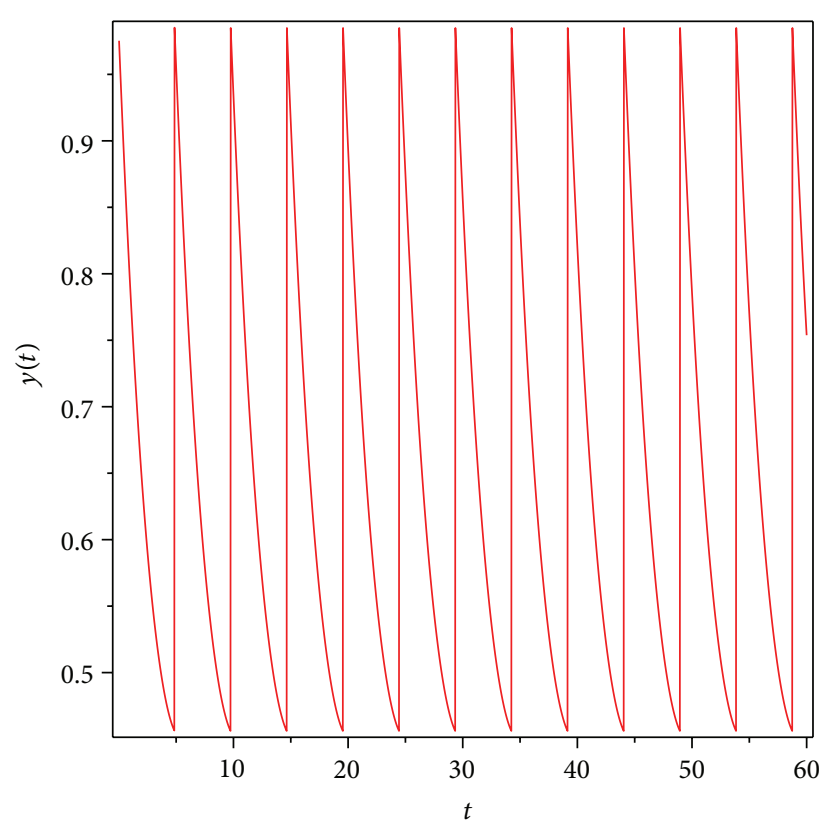

(c) Time series of system (24)

FIGURE 22: Illustration of basic behavior of system (24).

$$
\begin{array}{lll}
\frac{\partial \alpha}{\partial x}=-p, & \frac{\partial \alpha}{\partial y}=0, & \frac{\partial \beta}{\partial x}=0, \\
\frac{\partial \beta}{\partial y}=-q, & \frac{\partial \Phi}{\partial x}=1, & \frac{\partial \Phi}{\partial y}=0 .
\end{array}
$$

Thus,

$$
\begin{aligned}
\Delta_{1} & =\frac{(1-q) f\left(\phi\left(T^{+}\right), \varphi\left(T^{+}\right)\right)}{f(\phi(T), \varphi(T))} \\
& =\left((1-q)\left[\frac{r \phi\left(T^{+}\right)}{1+a \phi\left(T^{+}\right)}-b \phi\left(T^{+}\right) \varphi\left(T^{+}\right)\right]\right)
\end{aligned}
$$




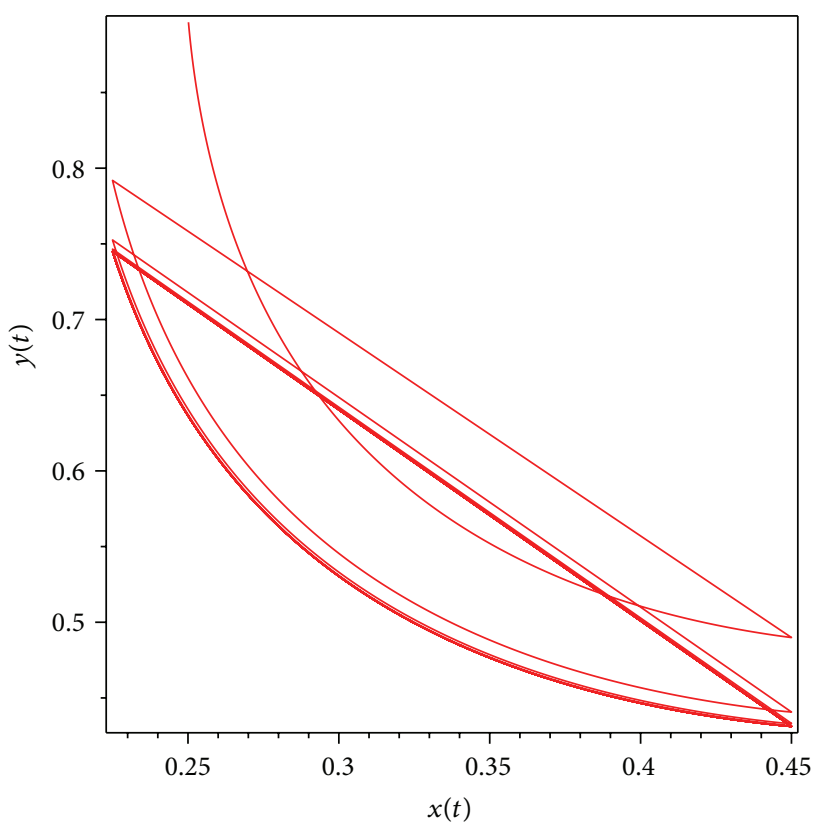

(a) Phase diagram of system (24)

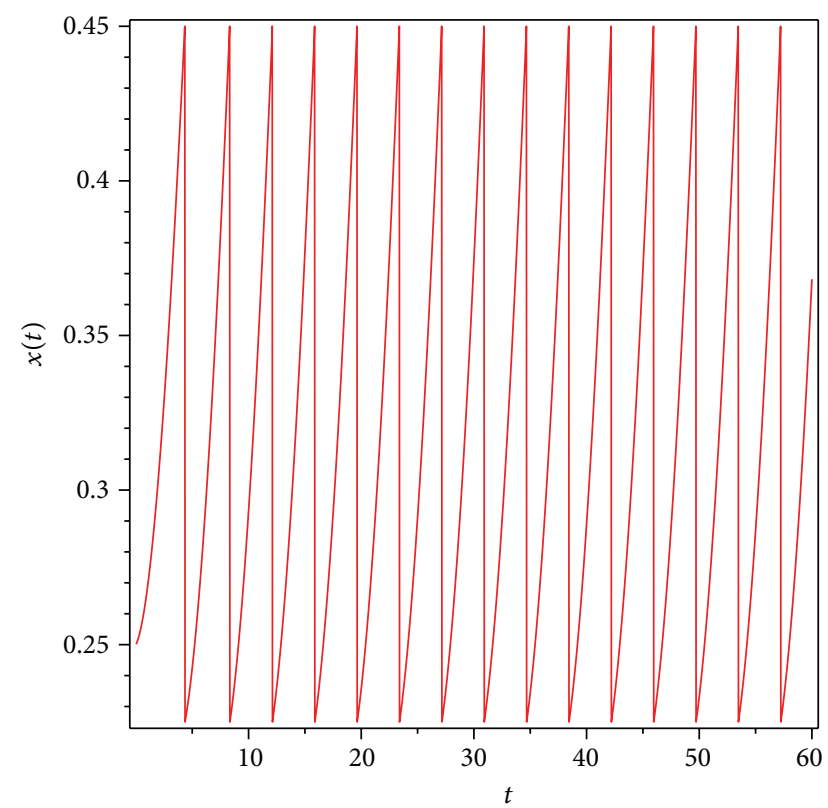

(b) Time series of system (24)

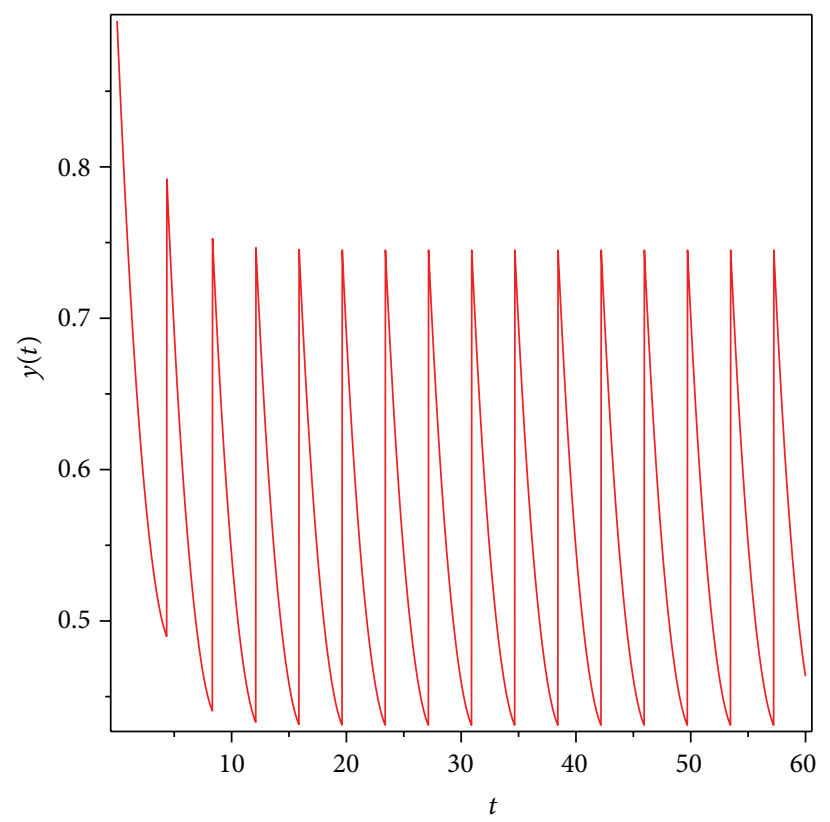

(c) Time series of system (24)

FIGURE 23: Illustration of basic behavior of system (24).

$$
\begin{aligned}
& \times\left(\frac{r \phi(T)}{1+a \phi(T)}-b \phi(T) \varphi(T)\right)^{-1} \\
= & \left((1-q)\left[\frac{r(1-p) h_{2}}{1+a(1-p) h_{2}}-b(1-p) h_{2} \varphi_{0}\right]\right) \\
& \times\left(\frac{r h_{2}}{1+a h_{2}}-b h_{2} \frac{\varphi_{0}-\tau}{1-q}\right)^{-1}
\end{aligned}
$$$$
=\left((1-p)(1-q)\left[\frac{r}{1+a(1-p) h_{2}}-b \varphi_{0}\right]\right)
$$$$
\times\left(\frac{r}{1+a h_{2}}-b \frac{\varphi_{0}-\tau}{1-q}\right)^{-1}
$$$$
\mu_{2}=\Delta_{1} \exp \int_{0}^{T}\left(\frac{\partial f}{\partial x}(\phi(t), \varphi(t))+\frac{\partial g}{\partial y}(\phi(t), \varphi(t))\right) d t
$$ 


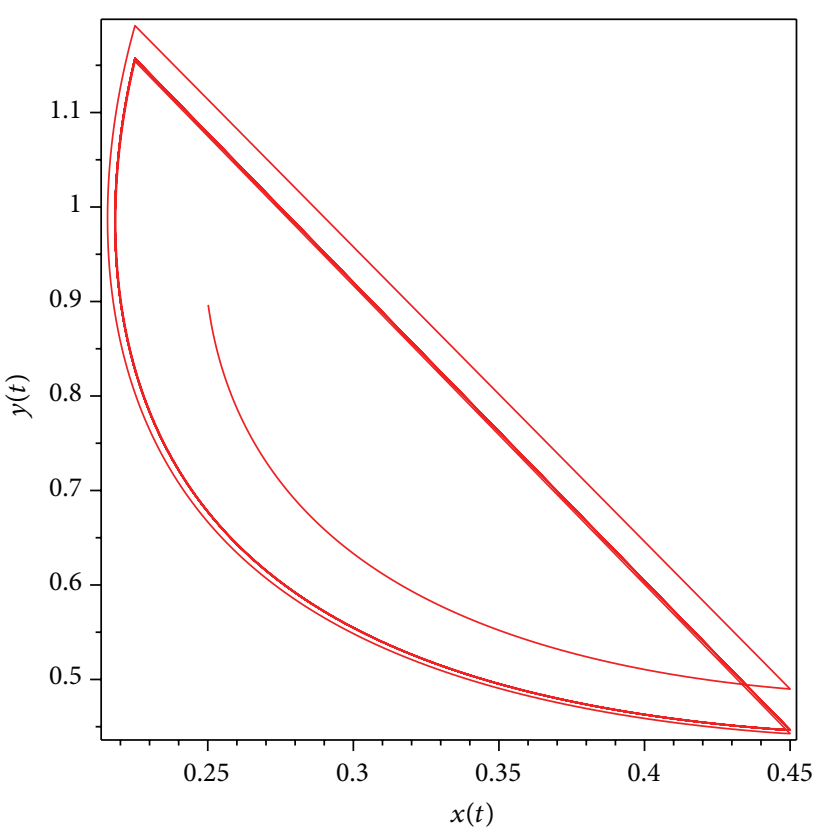

(a) Phase diagram of system (24)

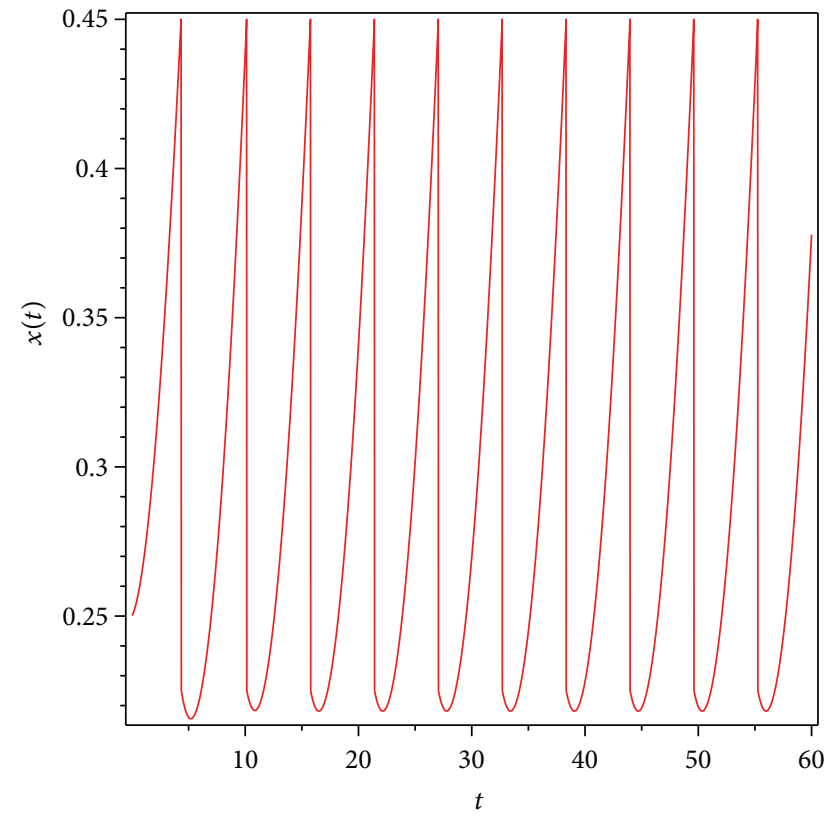

(b) Time series of system (24)

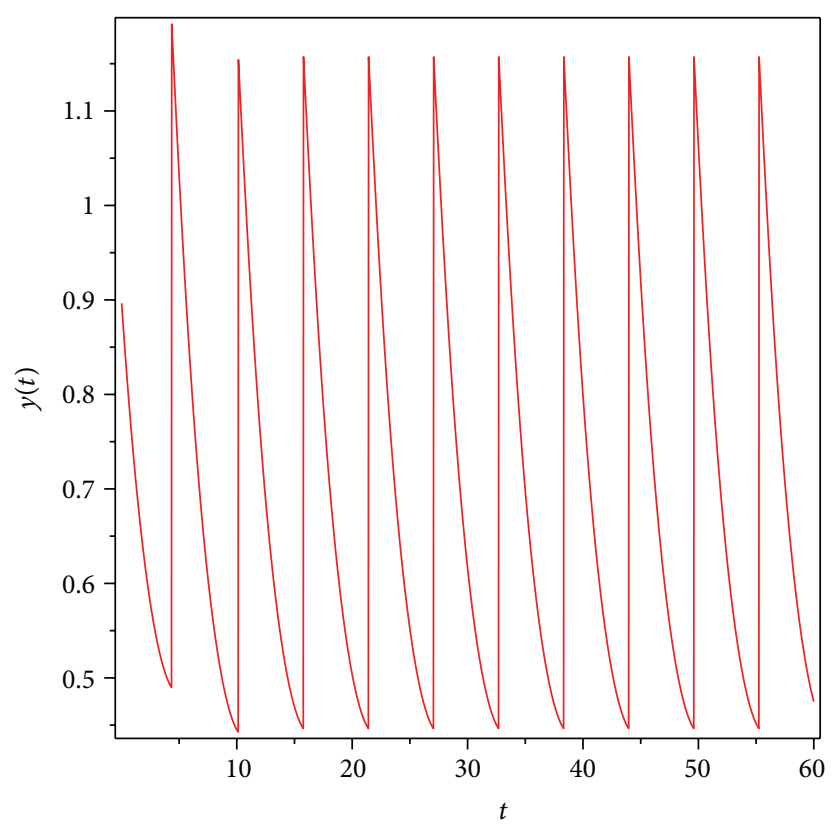

(c) Time series of system (24)

FIGURE 24: Illustration of basic behavior of system (24).

$$
\begin{array}{ll}
=\Delta_{1} \exp \int_{0}^{T}\left(\frac{r}{(1+a \phi)^{2}}-b \varphi+c \phi-d\right) d t & =\Delta_{1} \exp \left(\int_{0}^{T} \frac{1}{\phi(t)} d \phi(t)+\int_{0}^{T} \frac{1}{\varphi(t)} d \varphi(t)\right) \\
<\Delta_{1} \exp \int_{0}^{T}\left(\frac{r}{1+a \phi}-b \varphi+c \phi-d\right) d t & =\Delta_{1} \frac{1}{(1-q)(1-p)} \frac{\varphi_{0}-\tau}{\varphi_{0}}
\end{array}
$$




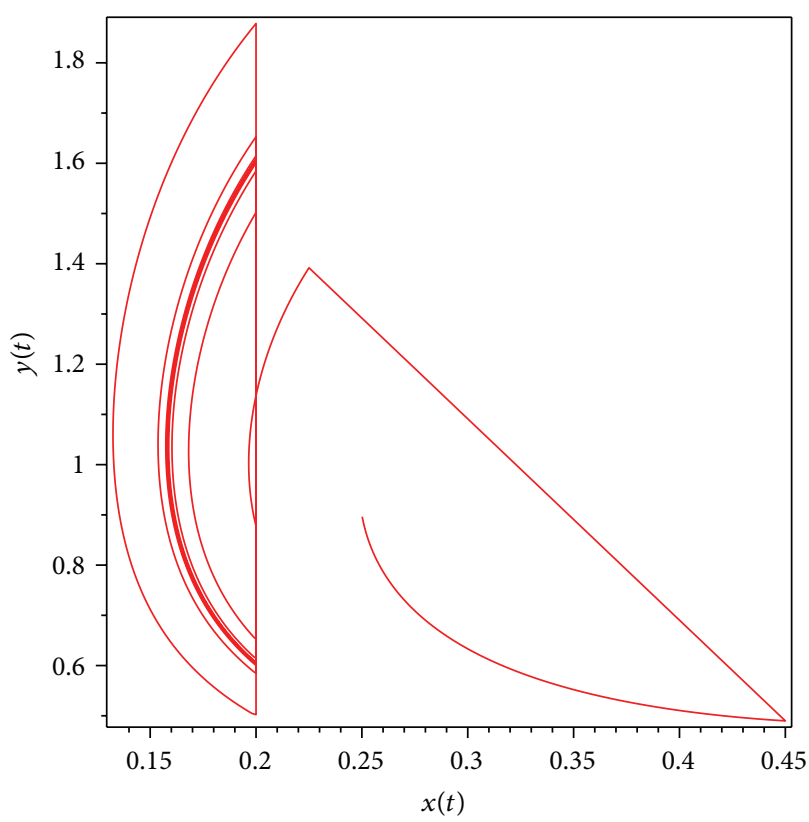

(a) Phase diagram of system (24)

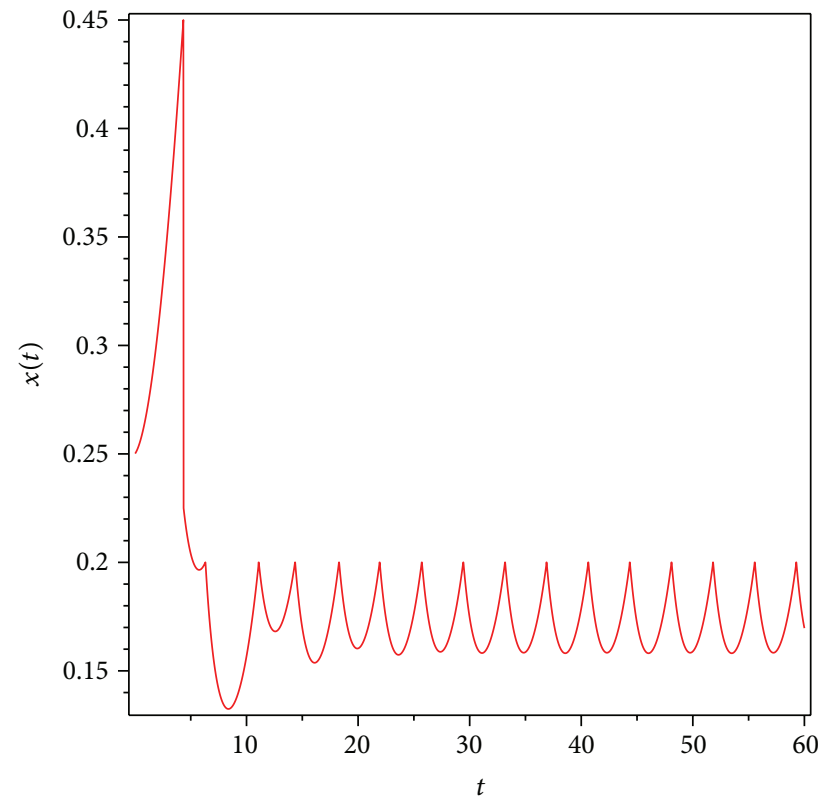

(b) Time series of system (24)

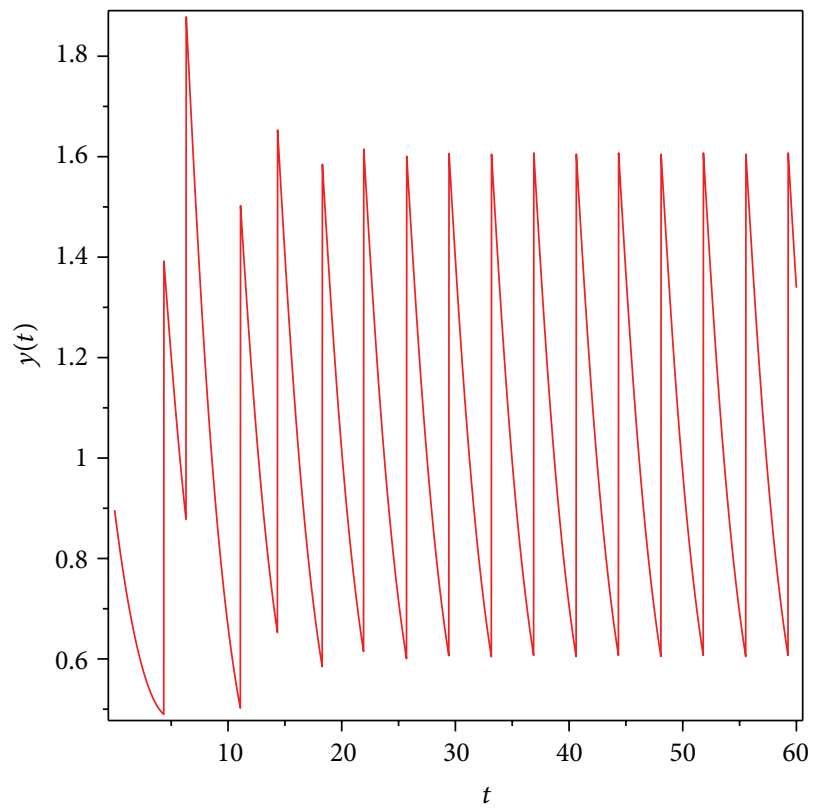

(c) Time series of system (24)

FIGURE 25: Illustration of basic behavior of system (24).

$$
\begin{array}{rlrl}
= & \frac{(1-p)(1-q)\left[\left(r /\left(1+a(1-p) h_{2}\right)\right)-b \varphi_{0}\right]}{\left(r /\left(1+a h_{2}\right)\right)-b\left(\left(\varphi_{0}-\tau\right) /(1-q)\right)} & \text { If } & \\
& \times \frac{1}{(1-q)(1-p)} \frac{\varphi_{0}-\tau}{\varphi_{0}} & \varphi_{0}<\min \left\{\frac{r(1-q)}{b\left(1+a h_{2}\right)}+\tau,\right. \\
\leq & \frac{\left(r /\left(1+a(1-p) h_{2}\right)\right)-b \varphi_{0}}{\left(r /\left(1+a h_{2}\right)\right)-b\left(\left(\varphi_{0}-\tau\right) /(1-q)\right)} . & \left.\frac{1}{b q}\left(b \tau-\frac{a p(1-q) r h_{2}}{\left(1+a h_{2}\right)\left(1+a(1-p) h_{2}\right)}\right)\right\}
\end{array}
$$


or

$$
\begin{aligned}
\varphi_{0}>\max \left\{\frac{r(1-q)}{b\left(1+a h_{2}\right)}+\tau,\right. \\
\\
\left.\qquad \frac{1}{b q}\left(b \tau-\frac{a p(1-q) r h_{2}}{\left(1+a h_{2}\right)\left(1+a(1-p) h_{2}\right)}\right)\right\},
\end{aligned}
$$

we have $\mu_{2}<1$; then the periodic solution to the system (1) is stable. This completes the proof.

\section{Example and Numerical Simulation}

To verify the validity of our results, we give an example. Let $r=0.6, a=1, b=0.5, c=0.8, d=0.4, p=0.5, q=0.2$, $h_{1}=0.2$, and $h_{2}=0.45$. By calculation, we obtain $y^{*}=1$, $P(0.2,1), Q(0.225,0.9796)$, and $E\left(x^{N}, y^{\aleph}\right)=(0.5,0.8)$. Then we have

$$
\begin{aligned}
& x^{\prime}(t)=\frac{0.6 x}{1+x}-0.5 x y, \quad x \neq 0.2,0.45 \text {, or } x=0.2, y>1 \text {, } \\
& y^{\prime}(t)=0.8 x y-0.4 y \\
& \Delta x=0, \\
& \Delta y=\alpha, \quad x=0.2, \quad y \leq 1, \\
& \Delta x=-0.5 x, \\
& \Delta y=-0.2 y+\tau, \quad x=0.45 \text {. }
\end{aligned}
$$

Numerical analysis of system (24) is being done using Maple 14.0. We have the following cases.

Case 1. Initial point $C_{0}$ is in the line $N_{1}$, where $\alpha=0.5$.

(a) Let $C_{0}$ be $(0.2,1.2)$ above $P(0.2,1)$ (see Figure 17).

(b) Let $C_{0}$ be $(0.2,0.6)$ below $P(0.2,1)$ (see Figure 18 ).

Case 2. Initial point $C_{0}$ is in the line $N_{2}$. Let $C_{0}$ be $Q(0.225,0.9796)$.

(a) The impulsive point $C_{1}^{+}$corresponding to $C_{1}$ is exactly $Q$, where $\tau=0.62$ (see Figure 19).

(b) The impulsive point $C_{1}^{+}$corresponding to $C_{1}$ is above Q on $N_{2}$, where $\tau=0.8$ (see Figure 20).

(c) The impulsive point $C_{1}^{+}$corresponding to $C_{1}$ is below $Q$ on $N_{2}$, where $\tau=0.5$ (see Figure 21).

Case 3. Initial point $C_{0}$ is between the second impulsive set $M_{2}$ and its image set $N_{2}$. Let $C_{0}$ be $(0.25,0.9)$.

(a) The impulsive point $C_{1}^{+}$corresponding to $C_{1}$ is exactly $Q$, where $\tau=0.62$ (see Figure 22).

(b) The impulsive point $C_{1}^{+}$corresponding to $C_{1}$ is below Q on $N_{2}$, where $\tau=0.4$ (see Figure 23).

(c) (1) The impulsive point $C_{1}^{+}$corresponding to $C_{1}$ is above $Q$ on $N_{2}$, where $\tau=0.8$ (see Figure 24).
(2) The impulsive point $C_{1}^{+}$corresponding to $C_{1}$ is above $Q$ on $N_{2}$, where $\tau=1$ and $\alpha=0.5$ (see Figure 25).

All the simulations show agreement with the results in this paper.

\section{Conclusion}

Certainly, the actual pest management will be very complicated; such biological models may be described by more state dependent pulses and time impulse, but they are difficult to study by the use of successor functions. In this paper, a pest management model with saturated growth rate is constructed. We proposed two state dependent impulsive effects in the model according to quantity of pests and natural enemies. Our primary results are to explain the effects of human interference in the actual pest management on dynamics of the system. If the quantity of pests is less than $h_{1}$, but the quantity of natural enemies is enough relatively $\left(>y^{*}\right)$, human interference is unnecessary. If the quantity of pests reaches a certain amount $h_{1}$, but the quantity of natural enemies is less than or equal to $y^{*}$, only biological control (releasing natural enemy) is implemented, and if the quantity of pests is large (reach $h_{2}$ ), integrated pest control (spraying pesticide and releasing natural enemy) is applied. We think our results will offer help to the actual pest management.

\section{Acknowledgments}

This work is supported by the Shandong Provincial Natural Sciences Funds of China (Grant no. ZR2012AM012) and a Project of Shandong Province Higher Educational Science and Technology Program of China (Grant no. J13LI05).

\section{References}

[1] "Canadian Forest Service," http://cfs.nrcan.gc.ca/home.

[2] S. M. Cook, Z. R. Khan, and J. A. Pickett, "The use of pushpull strategies in integrated pest management," Annual Review of Entomology, vol. 52, pp. 375-400, 2007.

[3] N. C. Elliott, J. A. Farrell, A. P. Gutierrez et al., Integrated Pest Management, Springer, New York, NY, USA, 1995.

[4] M. Kogan, "Integrated pest management: historical perspectives and contemporary developments," Annual Review of Entomology, vol. 43, pp. 243-270, 1998.

[5] C. Dai, M. Zhao, and L. Chen, "Homoclinic bifurcation in semi-continuous dynamic systems," International Journal of Biomathematics, vol. 5, no. 6, Article ID 1250059, 2012.

[6] Z. Li, L. Chen, and J. Huang, "Permanence and periodicity of a delayed ratio-dependent predator-prey model with Holling type functional response and stage structure," Journal of Computational and Applied Mathematics, vol. 233, no. 2, pp. 173-187, 2009.

[7] B. Liu, L. Chen, and Y. Zhang, "The dynamics of a preydependent consumption model concerning impulsive control strategy," Applied Mathematics and Computation, vol. 169, no. 1, pp. 305-320, 2005.

[8] X. Meng, J. Jiao, and L. Chen, "The dynamics of an age structured predator-prey model with disturbing pulse and time 
delays," Nonlinear Analysis. Real World Applications. An International Multidisciplinary Journal, vol. 9, no. 2, pp. 547-561, 2008.

[9] X. Song, M. Hao, and X. Meng, "A stage-structured predatorprey model with disturbing pulse and time delays," Applied Mathematical Modelling, vol. 33, no. 1, pp. 211-223, 2009.

[10] S. Tang and L. Chen, "Modelling and analysis of integrated pest management strategy," Discrete and Continuous Dynamical Systems B, vol. 4, no. 3, pp. 759-768, 2004.

[11] H. Zhang, L. Chen, and J. J. Nieto, "A delayed epidemic model with stage-structure and pulses for pest management strategy," Nonlinear Analysis. Real World Applications. An International Multidisciplinary Journal, vol. 9, no. 4, pp. 1714-1726, 2008.

[12] T. Zhang, X. Meng, and Y. Song, "The dynamics of a highdimensional delayed pest management model with impulsive pesticide input and harvesting prey at different fixed moments," Nonlinear Dynamics, vol. 64, no. 1-2, pp. 1-12, 2011.

[13] T. Zhang, X. Meng, Y. Song et al., "Dynamical analysis of delayed plant disease models with continuous or impulsive cultural control strategies," Abstract and Applied Analysis, vol. 2012, Article ID 428453, 25 pages, 2012.

[14] L. Nie, Z. Teng, L. Hu, and J. Peng, "Existence and stability of periodic solution of a predator-prey model with statedependent impulsive effects," Mathematics and Computers in Simulation, vol. 79, no. 7, pp. 2122-2134, 2009.

[15] S. Tang and R. A. Cheke, "State-dependent impulsive models of integrated pest management (IPM) strategies and their dynamic consequences," Journal of Mathematical Biology, vol. 50, no. 3, pp. 257-292, 2005.

[16] L. Nie, J. Peng, Z. Teng, and L. Hu, "Existence and stability of periodic solution of a Lotka-Volterra predator-prey model with state dependent impulsive effects," Journal of Computational and Applied Mathematics, vol. 224, no. 2, pp. 544-555, 2009.

[17] L. Nie, Z. Teng, L. Hu, and J. Peng, "Qualitative analysis of a modified Leslie-Gower and Holling-type II predator-prey model with state dependent impulsive effects," Nonlinear Analysis. Real World Applications. An International Multidisciplinary Journal, vol. 11, no. 3, pp. 1364-1373, 2010.

[18] L. Zhao, L. Chen, and Q. Zhang, "The geometrical analysis of a predator-prey model with two state impulses," Mathematical Biosciences, vol. 238, no. 2, pp. 55-64, 2012.

[19] D. Bainov and P. S. Simeonov, Impulsive Differential Equations: Periodic Solutions and Applications, Longman Scientific \& Technical, Harlow, UK, 1993.

[20] V. Lakshmikantham, D. D. Bainov, and P. S. Simeonov, Theory of Impulsive Differential Equations, World Scientific, Teaneck, NJ, USA, 1989. 


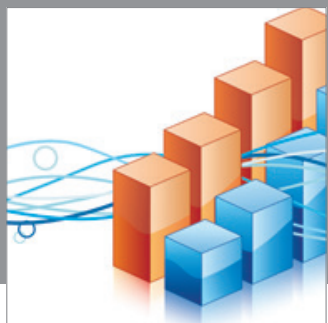

Advances in

Operations Research

mansans

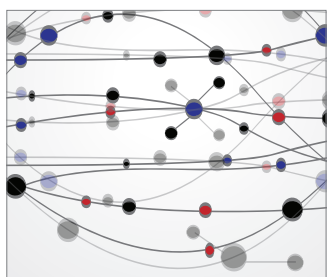

The Scientific World Journal
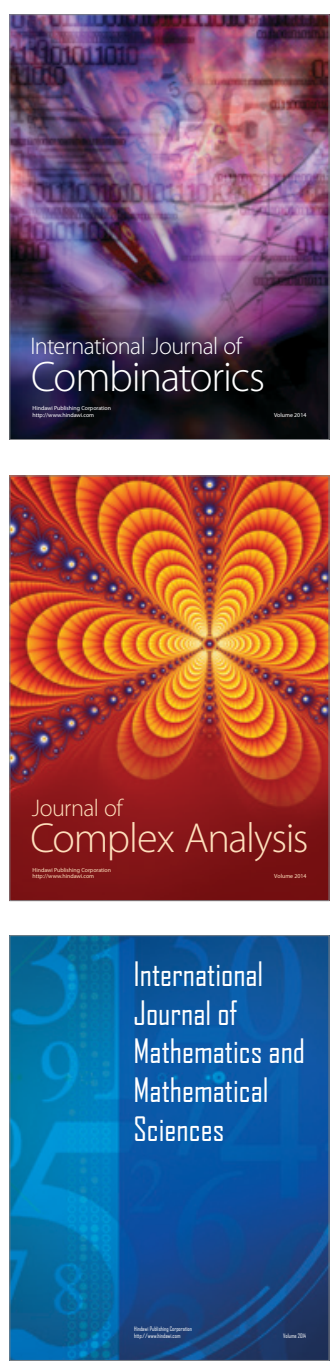
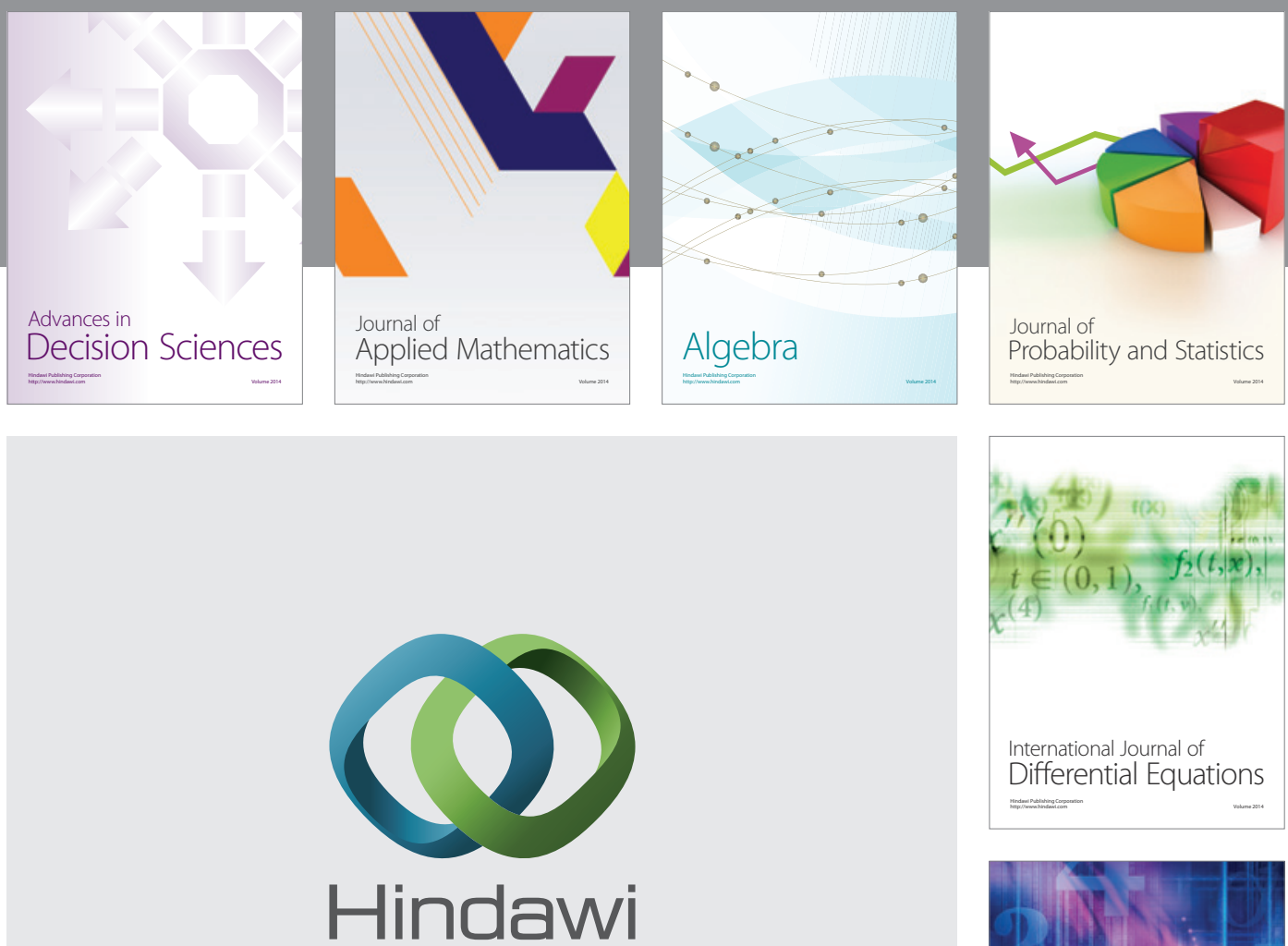

Submit your manuscripts at http://www.hindawi.com
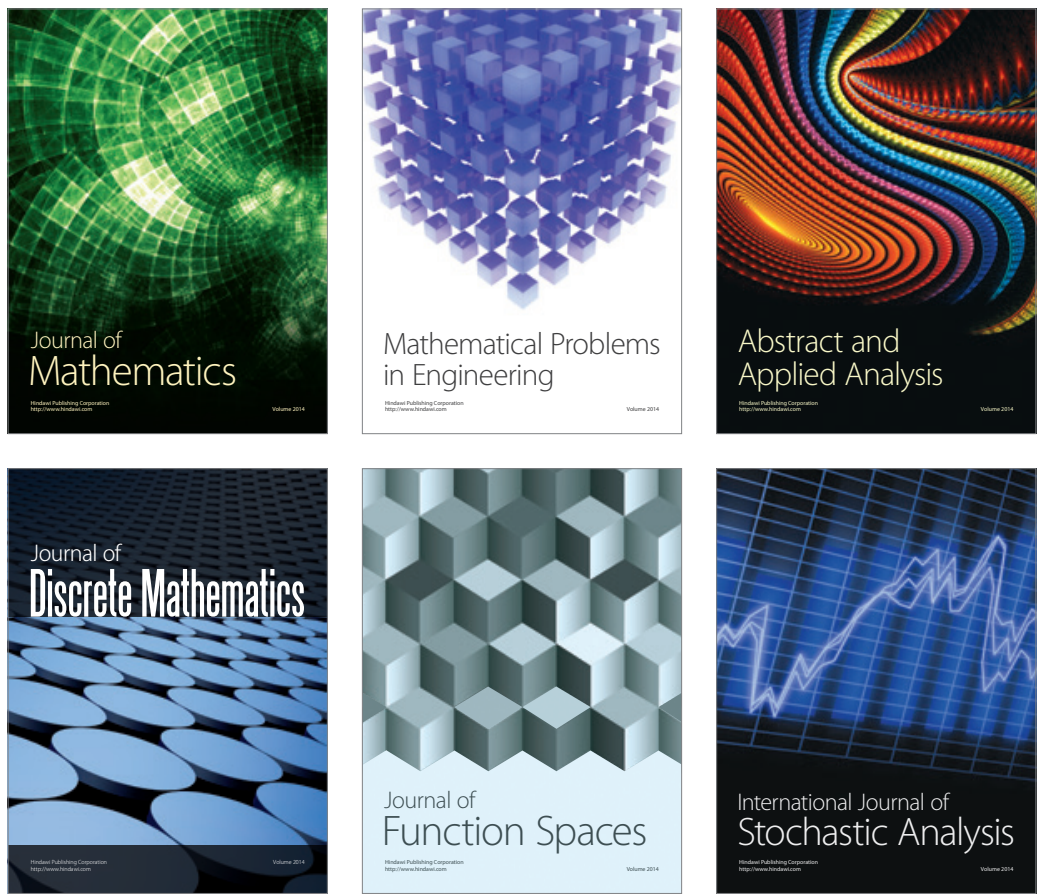

Journal of

Function Spaces

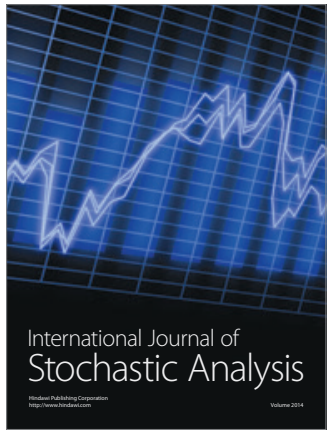

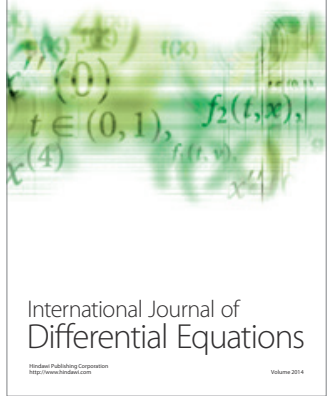
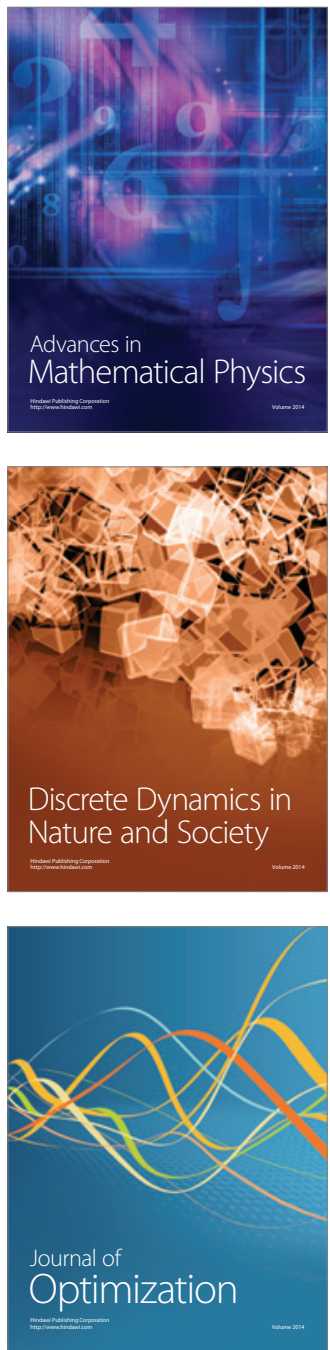